\title{
DIRICHLET INTERNAL LAYERS \\ IN AN EXCITABLE REACTION-DIFFUSION SYSTEM
}

\author{
Kunimochi Sakamoto
}

\begin{abstract}
In this paper we consider a singularly perturbed reaction-diffusion system of excitable media type with Dirichlet boundary conditions. We show that there exist multiple equilibrium solutions with internal transition and/or boundary layers. We also study the stability properties of these solutions.
\end{abstract}

\section{Introduction}

Chemical reactions with diffusive transport effects (Gray et al. [1990]), the development of biological structures (Murray [1989]), and many other pattern formation phenomena in nature have been studied by using reaction-diffusion equation models. In such models, the interaction and the subtle balance between nonlinear reaction kinetics and diffusive effects are responsible for a variety of spatio-temporal pattern formations. When a system is described by a two-component model of reactiondiffusion equations, we frequently encounter situations in which the diffusion rate of one (chemical, biological, or some other kind of) species is much smaller than that of the other. In such circumstances, singular perturbation methods provide us with a powerful tool to mathematically understand pattern formation mechanisms.

In this paper, we consider the following 1-dimensional reaction-diffusion equations on a finite interval

$$
\begin{aligned}
& u_{t}=\epsilon^{2} u_{x x}+f(u, v) \\
& v_{t}=v_{x x}+g(u, v)
\end{aligned} \quad t>0, \quad x \in(-l, l)
$$

with the Dirichlet boundary conditions

$$
u( \pm l, t)=v( \pm l, t)=0
$$

where $\epsilon>0$ is a small parameter and $l>0$ measures the size of the spatial domain. Both of these are adjustable parameters. The reaction kinetics $(f, g)$ is of excitable media type. A typical and suggestive example we have in mind is

$$
\begin{array}{llrl}
f(u, v) & =u(1-u)(u-a)-v, & & 0<a<1 / 2, \\
g(u, v) & =u-\gamma v, & & \gamma>0 \text { small, }
\end{array}
$$

which has been widely used as a prototype of (1.1).

The system (1.1) has been studied rather extensively either on an infinite spatial domain or on finite intervals with Neumann boundary conditions. Under such circumstances, the system (1.1) is known to have nonconstant equilibrium solutions with internal transition layers in the $u$-component (see Fife [1977] and Mimura, et al.

Received November 10, 1993, revised June 15, 1994.

Key words and phrases: transition layer, reaction-diffusion equations, singular perturbation, stability. 
[1980]). These solutions are constructed by using two stable branches of equilibrium solutions $u=h_{ \pm}(v)$ (see (H2) below) of the ordinary differential equation $u_{t}=f(u, v)$ and internal transition layers connecting the stable branches. Therefore, one can expect that the stability of these solutions is mainly determined by the local effect of the reaction-diffusion field. In fact, Nishiura and Fujii [1987] and Sakamoto [1990] rigorously worked out the spectral analysis to determine the stability of such solutions and gave a local condition which ensures the stability of these solutions. The stability of such solutions, as well as the stability analysis itself, is extremely delicate as was shown by Nishiura and Fujii [1987]. Namely, the critical eigenvalues which determine the stability are very small (of order $O(\epsilon)$ ) and the spectral analysis requires us to analyse a system of second-order differential operators with vanishing second derivative and discontinuous potential. Despite the subtlety of the stability and the stability analysis involved, it had been believed that the boundary conditions imposed on the system would not have much effect on the stability of the solutions with internal transition layers.

Motivated by the above, the purpose of this paper is to study whether or not equilibrium solutions with internal layers are stable under the Dirichlet boundary conditions. We will show that the system (1.1) has a singularly perturbed equilibrium solution with two internal layers (see Figure 4). This solution, however, turns out to be unstable under the Dirichlet boundary condition (1.2). Although the method of construction of such solutions as above has been standard in singular perturbation theories (Fife [1976]; Mimura, et al. [1980]), we will give an alternative way which employs the Liapunov-Schmidt method. The stability analysis presented in Sections 3 and 5 improves the presentation of the previous paper (Sakamoto [1990]), where the basic idea of the procedure was not stated clearly.

The result in the above cautions us to pay close attention to the boundary conditions under which a system is considered. Moreover, due to the development of open spatial reactors, a variety of experimental results exhibiting spatio-temporal patterns have been reported in chemistry; and reaction-diffusion models with the Dirichlet boundary conditions (reflecting the fact that the system is open) have been numerically, and theoretically to some extent, confirmed realistic and suitable to account for the phenomena observed (Elezgaray and Arneodo [1991]). Therefore, our problem $(1.1),(1.2)$ is not only of mathematical interest, but also will be a stepping stone to understand differences between open systems (Dirichlet or third boundary conditions) and closed systems (Neumann boundary conditions or in infinite spatial domains). Dirichlet boundary conditions also allow the system (1.1) to have patterns which can not exist in closed systems. In fact, we will construct solutions with boundary and interior transition layers and show that these are stable (see Figures 5 and 6).

In this paper, we assume the following conditions on $f$ and $g$ (see Figure 1):

(H-1) $\quad f$ and $g$ are smooth functions.

(H-2) The nullcline of $f$ consists of three pieces, $C_{+}, C_{-}, C_{0}$, where

$$
\begin{gathered}
C_{i}=\left\{(u, v) ; \quad u=h_{i}(v), \quad v \in I_{i}\right\}, \quad i=0,+,-, \\
I_{-}=\left(v_{0}, \infty\right), \quad I_{0}=\left(v_{0}, v_{1}\right), \quad I_{+}=\left(-\infty, v_{1}\right), \quad v_{0}<0<v_{1}
\end{gathered}
$$

and $h_{i}(v)(i=0,+,-)$ are smooth functions on $I_{i}$. The nullcline of $g$ intersects that of $f$ only at $(u, v)=(0,0)$ which lies on $C_{-}$. Moreover $g$ is positive on $C_{+}$and on $C_{0}$.

$$
f_{u}\left(h_{i}(v), v\right)<0, v \in I_{i}, i=+,- \text {. }
$$


(H-4) There exists a unique $v^{*} \in\left(0, v_{1}\right)$ such that $J\left(v^{*}\right)=0, J^{\prime}\left(v^{*}\right)<0$, where $J(v)$ is defined by $J(v)=\int_{h_{-}(v)}^{h_{+}(v)} f(s, v) d s$.

(H-5) $\quad g_{i}^{\prime}(v)<0, v \in I_{i}, i=+,-$, where $g_{i}(v)=g\left(h_{i}(v), v\right)$.

$(\mathrm{H}-6) \quad \int_{0}^{v^{*}} g_{-}(s) d s+\int_{v^{*}}^{v_{1}} g_{+}(s) d s>0$.

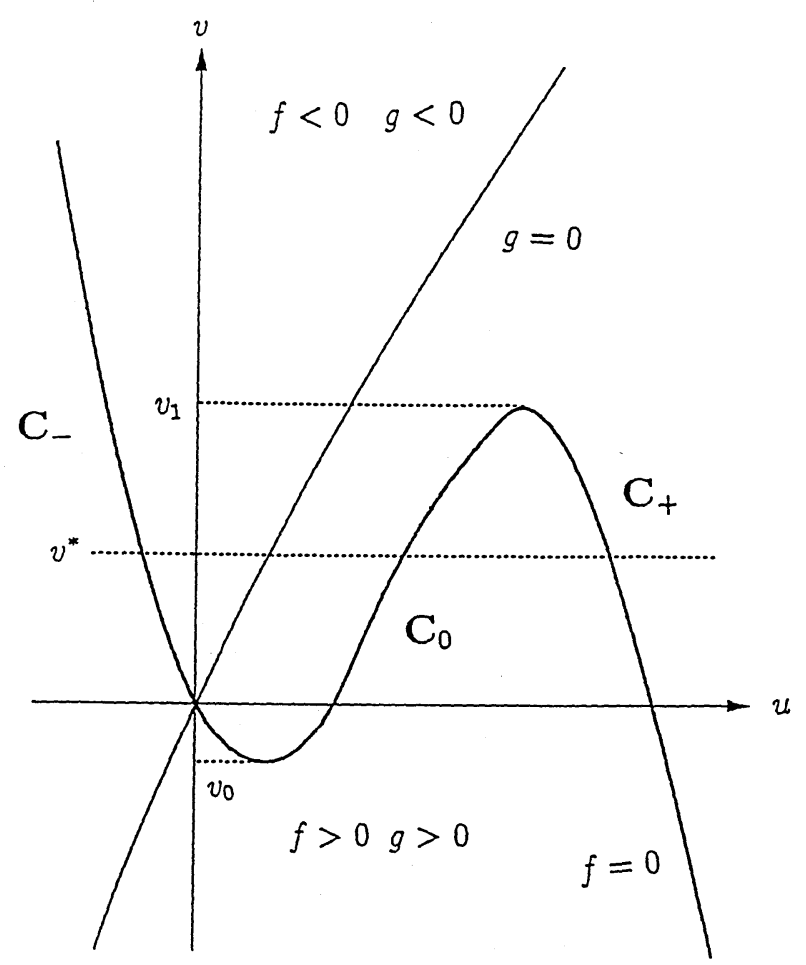

Figure 1. The nullcline of $(f, g)$.

Under these conditions, we will show that the problem $(1.1),(1.2)$ has steady state solutions with internal and boundary layers for small $\epsilon>0$. Two types of such solutions are constructed in this paper. The first type is a solution with two internal transition layers. This solution is symmetric around $x=0$. The second type is a solution with one internal layer and one boundary layer. There are two solutions of the second type, which are not symmetric around $x=0$, but one is the symmetric image of the other.

The equilibrium solutions of $(1.1),(1.2)$ satisfy

$$
0=\epsilon^{2} u_{x x}+f(u, v), \quad 0=v_{x x}+g(u, v), \quad u( \pm l)=0, \quad v( \pm l)=0 .
$$

In order to construct solutions of (1.3), we first solve the outer problem,

$$
v_{x x}+g_{*}(v)=0, x \in(-l, l), v( \pm l)=0, \quad g_{*}(v)= \begin{cases}g_{-}(v), & v \in\left[v_{0}, v^{*}\right] \\ g_{+}(v), & v \in\left(v^{*}, v_{1}\right]\end{cases}
$$

For problem (1.4), we have the following: 


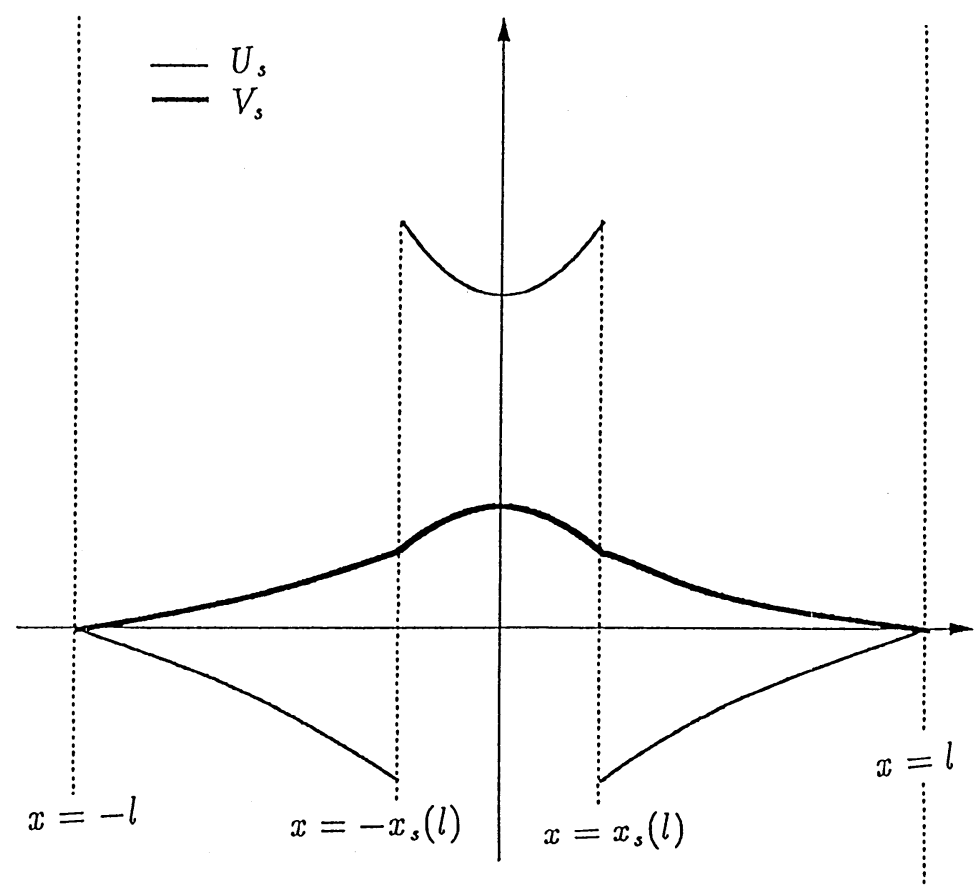

Figure 2. Profiles of symmetric outer solution $\left(U_{s}, V_{s}\right)$.

Theorem 1. Under the conditions $(\mathrm{H}-1)$ to $(\mathrm{H}-6)$ there exist a number $l_{0}>0$ and four functions,

$$
x_{s}(l)>0, x_{a}(l)>0, V_{s}(x, l), V_{a}(x, l), l \in\left[l_{0}, \infty\right), x \in[-l, l],
$$

such that

(i) $V_{s}$ is a solution of (1.4), $C^{1}$-matched at $\pm x_{s}(l)$, and satisfies

$$
\begin{array}{cl}
V_{x x}+g_{-}(V)=0, & -l<x<-x_{s}(l), \quad x_{s}(l)<x<l \\
V_{x x}+g_{+}(V)=0, \quad & -x_{s}(l)<x<x_{s}(l), \\
V( \pm l)=0, \quad V_{x}(0)=0, \quad V\left( \pm x_{s}(l)\right)=v^{*}, \quad V(-x)=V(x)
\end{array}
$$

(ii) $V_{a}$ is a solution of (1.4), $C^{1}$-matched at $x_{a}(l)$, and satisfies

$$
\begin{aligned}
V_{x x}+g_{-}(V)=0, & -l<x<x_{a}(l) \\
V_{x x}+g_{+}(V)=0, & x_{a}(l)<x<l \\
V( \pm l)=0, & V\left(x_{a}(l)\right)=v^{*}
\end{aligned}
$$

(iii) The functions $x_{s}(l)$ and $x_{a}(l)$ are smooth in $l \in\left(l_{0},-\infty\right)$ and satisfy

$$
\begin{gathered}
\lim _{l \rightarrow \infty} x_{s}(l)=\int_{v^{*}}^{\beta_{0}} \frac{d v}{\sqrt{2 \int_{v}^{\beta_{0}} g_{+}(s) d s}} \\
\lim _{l \rightarrow \infty}\left(l-x_{a}(l)\right)=2 \lim _{l \rightarrow \infty} x_{s}(l)+\int_{0}^{v^{*}} \frac{d v}{\sqrt{2 \int_{v}^{\beta_{0}} g_{+}(s) d s}}
\end{gathered}
$$




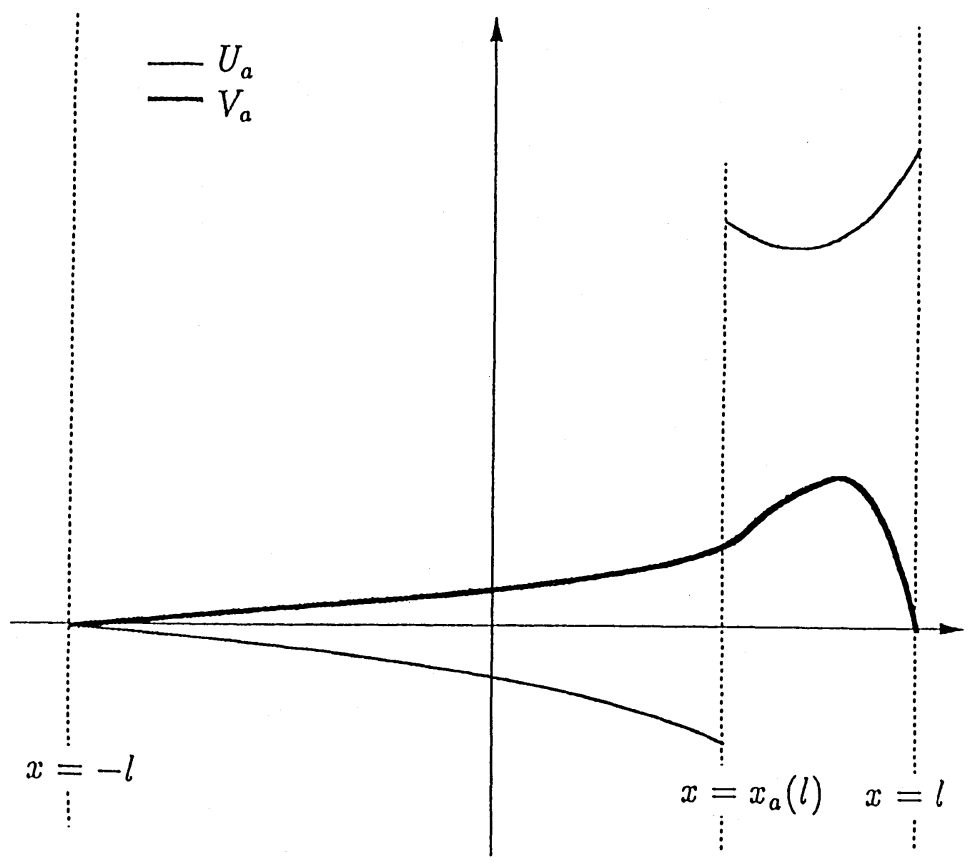

Figure 3. Profiles of antisymmetric outer solution $\left(U_{a}, V_{a}\right)$.

where $\beta_{0} \in\left(v^{*}, v_{1}\right)$ is a unique solution of

$$
\int_{0}^{v^{*}} g_{-}(s) d s+\int_{v^{*}}^{\beta} g_{+}(s) d s=0
$$

In terms of the functions $V_{s}$ and $V_{a}$, one defines so-called outer solutions of the problem (1.3) given by the pairs $\left(U_{s}, V_{s}\right)$, and $\left(U_{a}, V_{a}\right)$ (see Figures 2, 3), where

$$
U_{s}(x)=\left\{\begin{array}{ll}
h_{-}\left(V_{s}(x)\right), & x_{s}(l)<|x|<l, \\
h_{+}\left(V_{s}(x)\right), & |x|<x_{s}(l),
\end{array} \quad U_{a}(x)= \begin{cases}h_{-}\left(V_{a}(x)\right), & -l<x<x_{a}(l), \\
h_{+}\left(V_{a}(x)\right) & x_{a}(l)<x<l .\end{cases}\right.
$$

The function $U_{s}(x)$ is smooth except for two jump discontinuities at $x= \pm x_{s}(l)$ and satisfies the boundary conditions in (1.3). The function $U_{a}(x)$ is also smooth except for a jump discontinuity at $x=x_{a}(l)$ and does not satisfy the boundary condition in (1.3) at $x=l$. By taking advantage of the small parameter $\epsilon^{2}$ in front of the second derivative $u_{x x}$ in (1.3), one can construct two solutions of (1.3) for small $\epsilon>0$. One solution has two internal transition layers of width $O(\epsilon)$ near $x= \pm x_{s}(l)$, and the other has one internal transition layer of width $O(\epsilon)$ near $x=x_{a}(l)$ and one boundary layer at $x=l$. These two solutions are close, in a sense specified later, to the outer solutions $\left(U_{s}(x), V_{s}(x)\right)$ and $\left(U_{a}(x), V_{a}(x)\right)$, respectively. More precisely, we have the following theorem (see Figures 4, 5, and 6). 
Theorem 2. Under the conditions ( $\mathrm{H}-1)$ to $(\mathrm{H}-6)$, there is a constant $\epsilon_{0}>0$ such that for each $l>l_{0}$, the problem (1.1), (1.2) has two $\epsilon$-families of equilibrium solutions $\left(u_{s}^{\epsilon}, v_{s}^{\epsilon}\right)$ and $\left(u_{a}^{\epsilon}, v_{a}^{\epsilon}\right)$ for $\epsilon \in\left(0, \epsilon_{0}\right]$ satisfying

(i)

$$
\lim _{\epsilon \rightarrow 0}\left|v_{j}^{\epsilon}(x)-V_{j}(x)\right|=0
$$

uniformly on $[-l, l]$ for $j=s, a$;

(iis)

$$
\lim _{\epsilon \rightarrow 0}\left|u_{s}^{\epsilon}(x)-U_{s}(x)\right|=0
$$

uniformly on any compact sets of $\left[-l,-x_{s}(l)\right) \cup\left(-x_{s}(l), x_{s}(l)\right) \cup\left(x_{s}(l), l\right]$;

(iia)

$$
\lim _{\epsilon \rightarrow 0}\left|u_{a}^{\epsilon}(x)-U_{a}(x)\right|=0
$$

uniformly on any compact sets of $\left[-l, x_{a}(l)\right) \cup\left(x_{a}(l), l\right)$;

(iii) for each $\delta>0$ small, the set

$$
\left\{x \in[-l, l] ; \quad\left|u_{j}^{\epsilon}(x)-U_{j}(x)\right| \geq \delta\right\}
$$

consists of intervals of width $O(\epsilon)$ as $\epsilon \rightarrow 0$ near $x= \pm x_{s}(l)$ (for $j=s$ ) or near $x=x_{a}(l)$ and $x=l($ for $j=a)$.

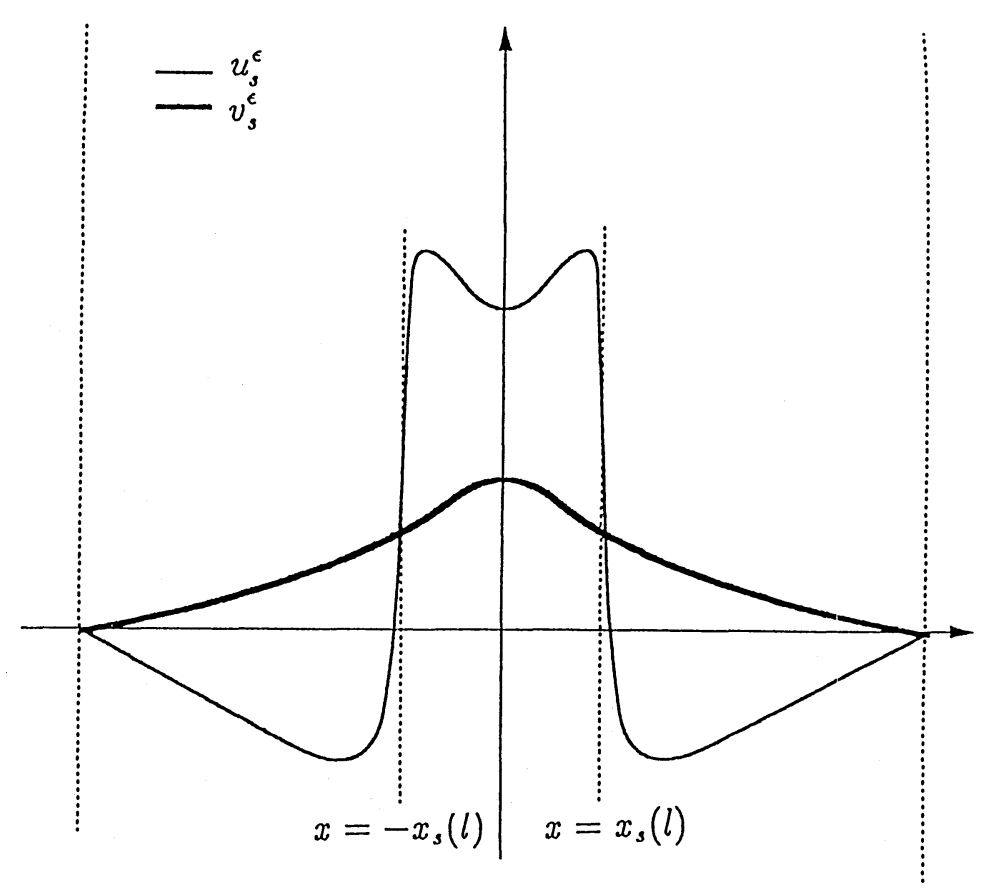

Figure 4. Profiles of symmetric solution $\left(u_{s}^{\epsilon}, v_{s}^{\epsilon}\right)$. 


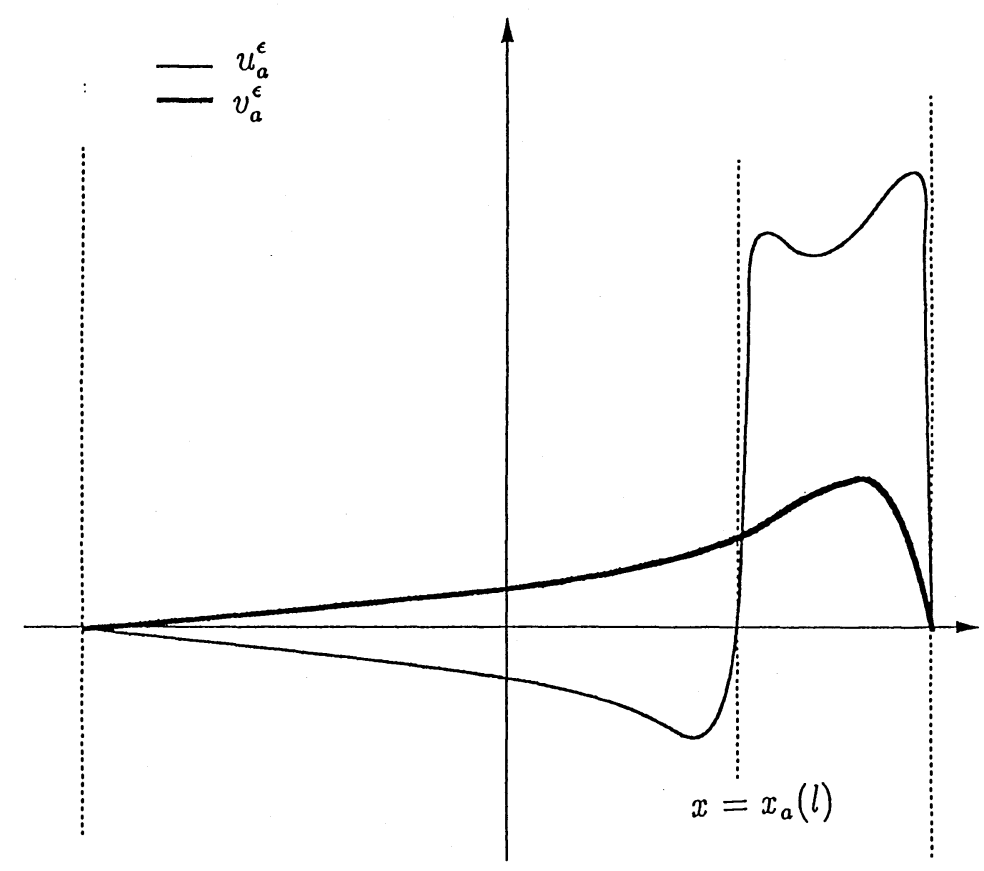

Figure 5. Profiles of antisymmetric solution $\left(u_{a}^{\epsilon}, v_{a}^{\epsilon}\right)$.

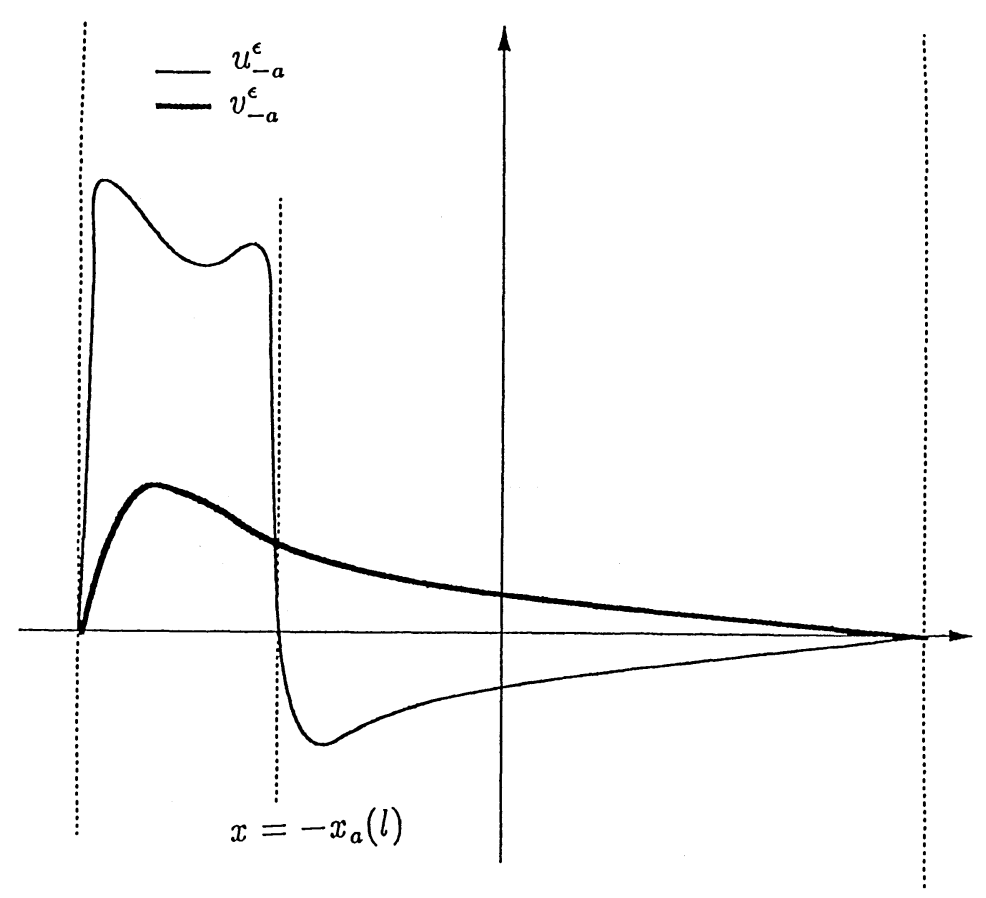

FiguRE 6. Profiles of antisymmetric solution $\left(u_{-a}^{\epsilon}, v_{-a}^{\epsilon}\right)$. 
As for the stability properties of the solutions $\left(u_{s}^{\epsilon}, v_{s}^{\epsilon}\right)$ and $\left(u_{a}^{\epsilon}, v_{a}^{\epsilon}\right)$, we have the following result.

Theorem 3. (i) For $\epsilon>0$ small, the solution $\left(u_{s}^{\epsilon}, v_{s}^{\epsilon}\right)$ is an unstable steady state solution of the parabolic system (1.1),(1.2). More precisely, there is a constant $\rho_{0}>0$ such that the eigenvalue problem associated with the linearization of (1.1),(1.2) around $\left(u_{s}^{\epsilon}, v_{s}^{\epsilon}\right)$ has exactly two simple, real eigenvalues $\rho_{s}^{\epsilon}$ and $\rho_{a}^{\epsilon}$ in the region $\Re \rho>-\rho_{0}$, and the remaining part of the spectrum is contained in the region $\Re \rho \leq-\rho_{0}$. The two eigenvalues have the following asymptotic expressions:

$$
\rho_{s}^{\epsilon}=\epsilon\left(\hat{\rho_{s}}+o(1)\right), \quad \rho_{a}^{\epsilon}=\epsilon\left(\hat{\rho_{a}}+o(1)\right), \quad \text { as } \epsilon \rightarrow 0, \quad \hat{\rho_{s}}<0, \quad \hat{\rho_{a}}>0,
$$

and the corresponding eigenfunctions are symmetric and antisymmetric, respectively.

(ii) For $\epsilon>0$ small, the solution $\left(u_{a}^{\epsilon}, v_{a}^{\epsilon}\right)$ is a stable equilibrium solution of the parabolic system (1.1), (1.2). In the region $\Re \rho>-\rho_{0}$, there is exactly one simple, real eigenvalue of the eigenvalue problem associated with the linearization of (1.1), (1.2) around the solution $\left(u_{a}^{\epsilon}, v_{a}^{\epsilon}\right)$. This eigenvalue has the asymptotic expression $\rho^{\epsilon}=$ $\epsilon(\hat{\rho}+o(1))$ as $\epsilon \rightarrow 0, \hat{\rho}<0$, and the remaining part of the spectrum is contained in the region $\Re \rho \leq-\rho_{0}$.

If we denote by $\left(u_{-a}^{\epsilon}(x), v_{-a}^{\epsilon}(x)\right)$ the pair $\left(u_{a}^{\epsilon}(-x), v_{a}^{\epsilon}(-x)\right)$, then it is also an equilibrium solution of (1.1), (1.2) with the same stability property as $\left(u_{a}^{\epsilon}, v_{a}^{\epsilon}\right)$. Since the eigenfunction associated with the unstable eigenvalue of the symmetric solution $\left(u_{s}^{\epsilon}, v_{s}^{\epsilon}\right)$ is antisymmetric (which corresponds to translation in the limit $l \rightarrow \infty$ ), it is suggested that there are two orbits of $(1.1),(1.2)$ which connect $\left(u_{s}^{\epsilon}, v_{s}^{\epsilon}\right)$ with $\left(u_{a}^{\epsilon}, v_{a}^{\epsilon}\right)$ and $\left(u_{-a}^{\epsilon}, v_{-a}^{\epsilon}\right)$, respectively. See Figure $7 \mathrm{a}$, in which $W^{s}$ stands for the stable manifold of $\left(u_{s}^{\epsilon}, v_{s}^{\epsilon}\right)$. This is numerically confirmed, as the profiles in Figure $7 \mathrm{~b}$ show.

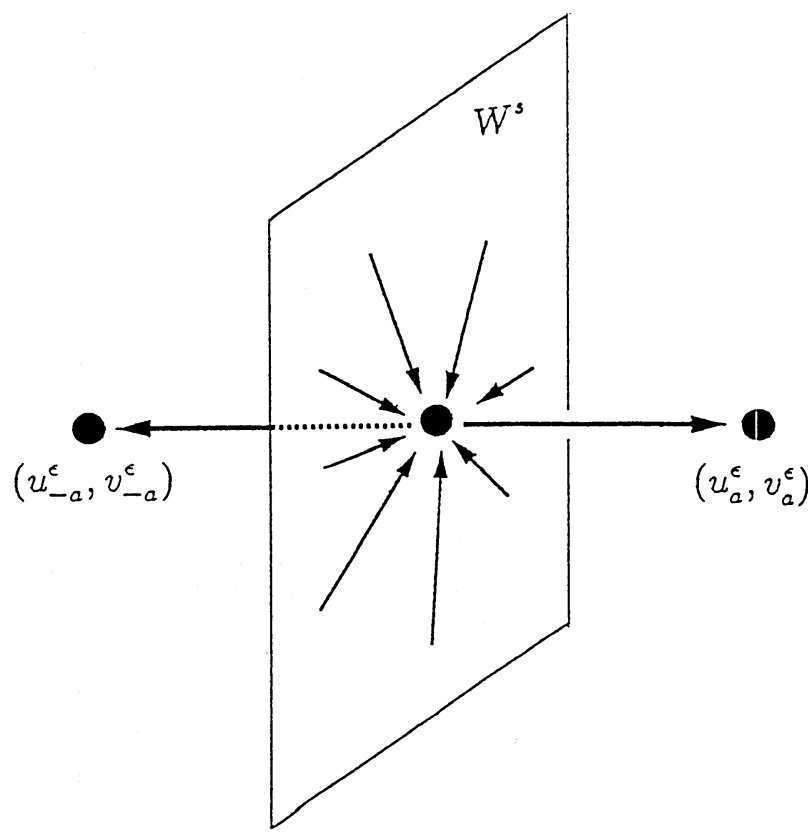

FIgURE 7a. Phase portrait near $\left(u_{s}^{\epsilon}, v_{s}^{\epsilon}\right)$ and $\left(u_{ \pm a}^{\epsilon}, v_{ \pm a}^{\epsilon}\right)$ 

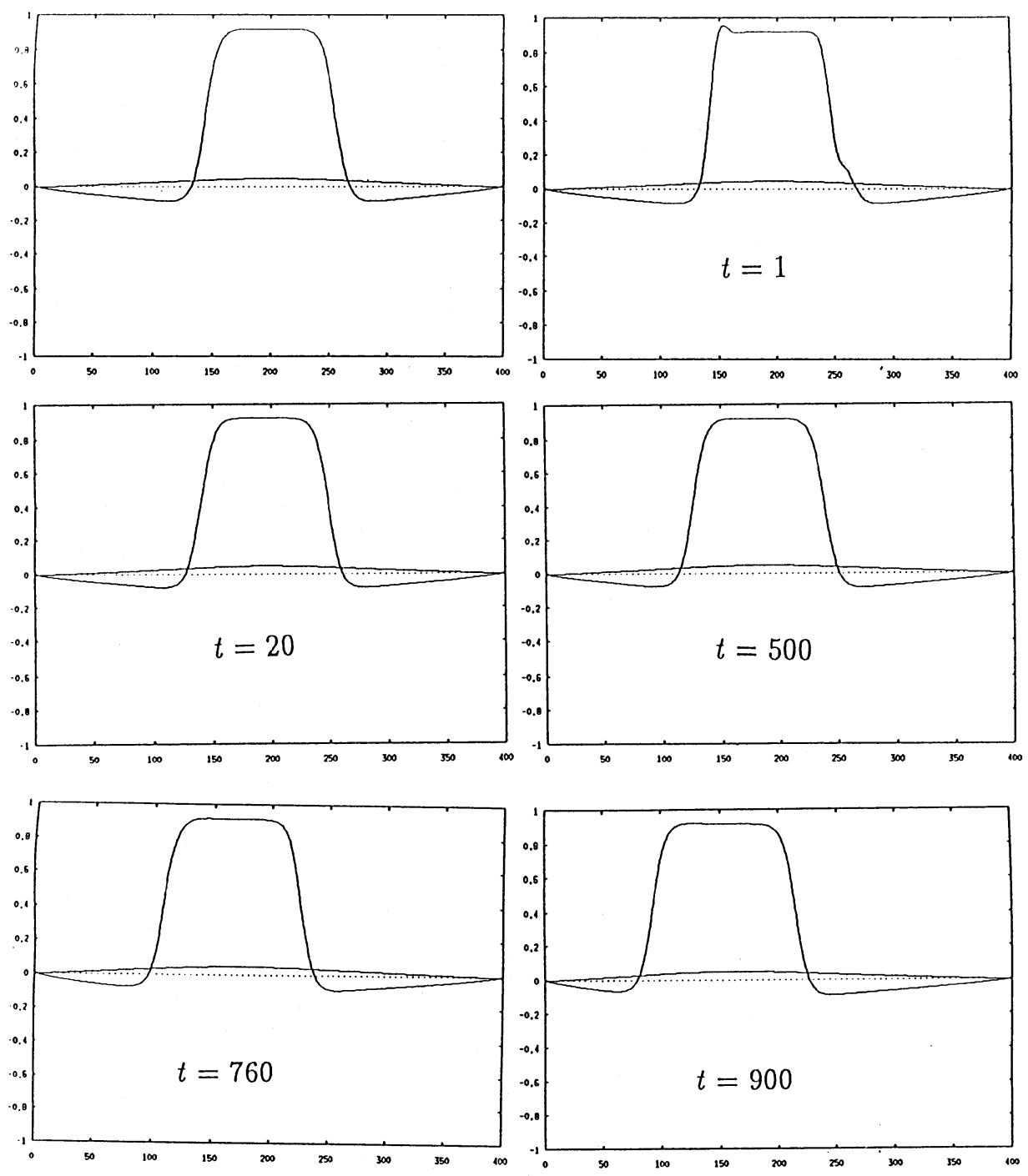

FigURE 7b. Numerical simulation of the orbit connecting $\left(u_{s}^{\epsilon}, v_{s}^{\epsilon}\right)$ with $\left(u_{-a}^{\epsilon}, v_{-a}^{\epsilon}\right)$. 

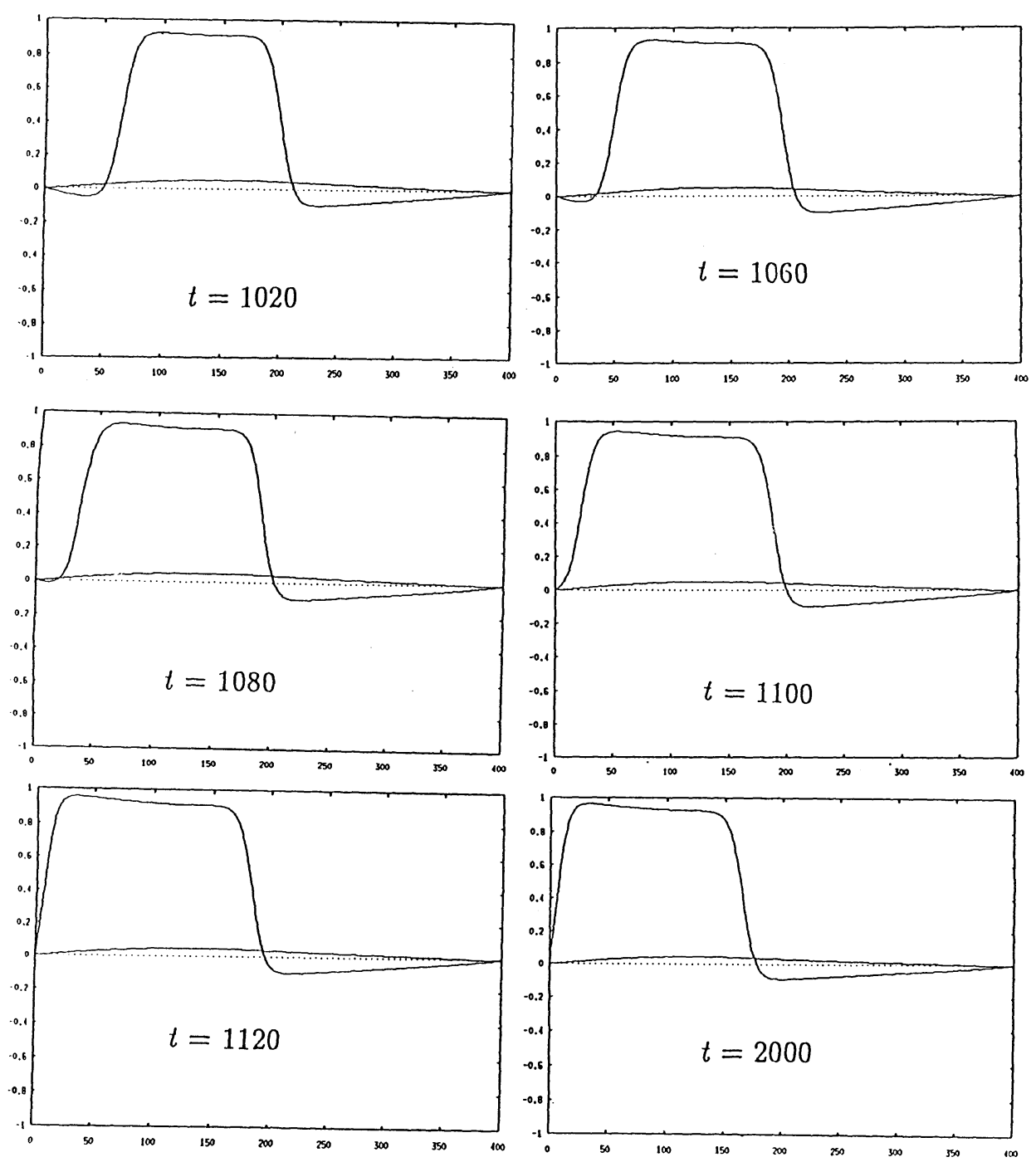

FigURE $7 \mathrm{~b}$ (continued). Numerical simulation of the orbit connecting $\left(u_{s}^{\epsilon}, v_{s}^{\epsilon}\right)$ with $\left(u_{-a}^{\epsilon}, v_{-a}^{\epsilon}\right)$. 
In this numerical simulation, we used the following nonlinearity

$$
\begin{aligned}
& f(u, v)=u(1-u)(u-a)-v, \\
& g(u, v)=u-\gamma v
\end{aligned}
$$

with $a=0.25, \gamma=0.25$, and the layer parameter $\epsilon=0.01$. The symmetric equilibrium solution in Figure $7 \mathrm{~b}$ was obtained by solving the evolution equation (1.1) with a symmetric initial function. Note that the symmetric equilibrium solution is stable under symmetric perturbations. The numerical integration was carried out until the profile settled down. We then added an antisymmetric perturbation to it and continued the numerical integration further. After a short period of initial relaxation, one can observe the profile with a well-defined layer structure moving slowly towards the left. As long as the position of the left layer is not close to the boundary, the profile of the solution is almost identical to a translation of the symmetric equilibrium solution. When the position of the left layer comes closer to the boundary, it speeds up and the internal layer quickly settles down to the boundary layer. At this stage, the position of the right layer is still moving slowly towards the left, and the entire profile eventually converges to that of the antisymmetric equilibrium solution $\left(u_{-a}^{\epsilon}, v_{-a}^{\epsilon}\right)$.

To understand the meaning of Theorem 3(i) better, let us consider the system (1.1) under the following boundary conditions

$$
\begin{aligned}
\alpha u(-l)-(1-\alpha) u_{x}(-l) & =0=\alpha v(-l)-(1-\alpha) v_{x}(-l), \\
\alpha u(l)+(1-\alpha) u_{x}(l) & =0=\alpha v(l)+(1-\alpha) v_{x}(l),
\end{aligned}
$$

where $0 \leq \alpha \leq 1$. When $\alpha=0$, the equilibrium solution with transition layers is stable (Nishiura and Fujii [1987]) and it is unstable when $\alpha=1$, which we have shown. Therefore, one may expect that there is a critical value $\alpha^{*} \in(0,1)$ where the stability property changes when $\alpha$ is varied. This statement turns out to be true, which will be reported in a future publication.

Throughout the paper, we use the following notation.

- $H^{0}=L^{2}[-l, 0], H^{j}=H^{j}[-l, 0], j=1,2$; the usual Sobolev space.

- $H_{\epsilon}^{2}=H^{2}[-l, 0]$ equipped with the weighted norm $\|u\|_{H_{\epsilon}^{2}}=\sum_{j=0}^{2}\left\|\epsilon^{j} d^{j} u / d x^{j}\right\|$.

- $H_{D N}^{2}=\left\{u \in H^{2} \mid u(-l)=0=u_{x}(0)\right\}$.

- $H_{D D}^{2}=\left\{u \in H^{2} \mid u(-l)=0=u(0)\right\}$.

- $H_{D N, \epsilon}^{2}=H_{D N}^{2}$ equipped with the weighted norm $\|\cdot\|_{H_{\epsilon}^{2}}$.

- $\langle u, v\rangle=\int_{-l}^{0} u(x) v(x) d x$, the $L^{2}$-inner product.

\section{Approximation}

In this section, we construct approximate solutions to the problem (1.3) which exhibit appropriate internal and/or boundary layers. The procedure in this section closely follows that of Ito [1985] and Sakamoto [1990]. See Mimura, et al. [1980] and Nishiura and Fujii [1987] for another treatment.

2.1. Outer solutions. Consider the phase plane of $v^{\prime \prime}+g_{*}(v)=0$ (see Figure 8). Let $l_{1}(\beta)$ be the "time" spent between $A$ and $B$, and $l_{2}(\beta)$ the "time" spent between $B$ and $C$ where $C=(\beta, 0)$. We have

$$
l_{1}(\beta)=\int_{0}^{v^{*}} \frac{d v}{\sqrt{2 \int_{v}^{v^{*}} g_{-}(s) d s+2 \int_{v^{*}}^{\beta} g_{+}(s) d s}}, \quad l_{2}(\beta)=\int_{v^{*}}^{\beta} \frac{d v}{\sqrt{2 \int_{v}^{\beta} g_{+}(s) d s}},
$$




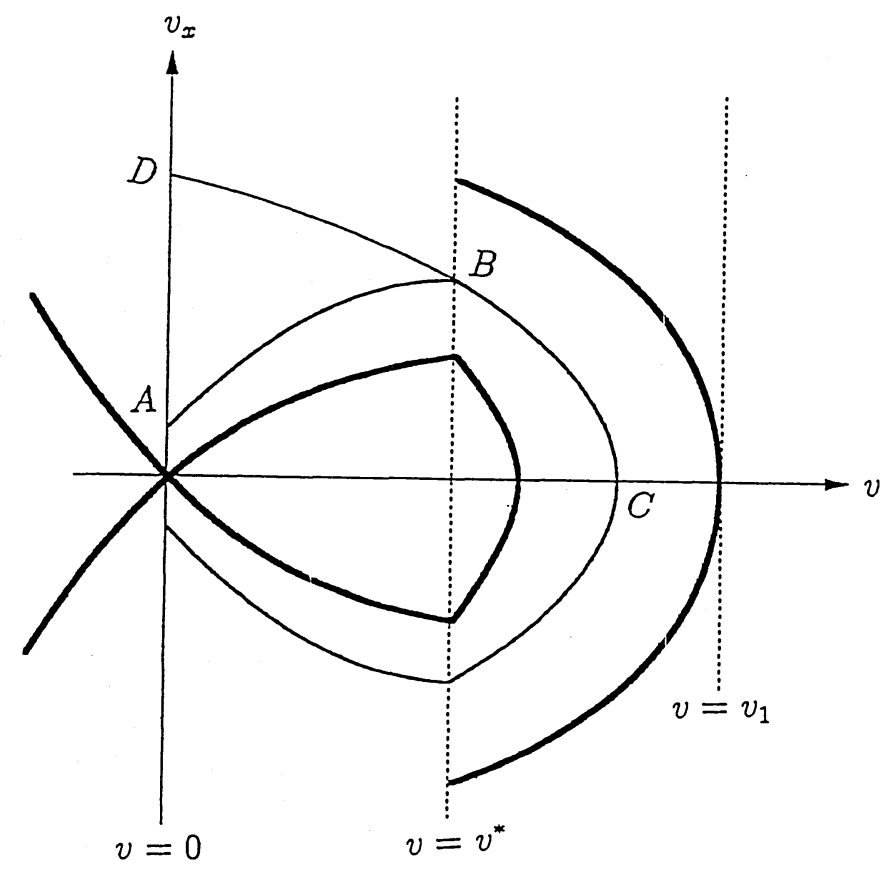

Figure 8. Phase plane of $v_{x x}+g_{*}(v)=0$.

for $\beta \in\left(\beta_{0}, v_{1}\right)$, where $\beta_{0}$ is defined in Theorem 1 (iii).

The desired solution $V_{s}$ can be determined by solving $l_{1}(\beta)+l_{2}(\beta)=l$. One can verify $l_{1}{ }^{\prime}(\beta)<0$ and $l_{2}{ }^{\prime}>0$ due to $g_{+}>0$ and $g_{+}{ }^{\prime}<0$. Moreover, one has

$$
\begin{array}{cc}
\lim _{\beta \rightarrow \beta_{0}} l_{1}(\beta)=\infty, & \lim _{\beta \rightarrow \beta_{0}} l_{1}^{\prime}(\beta)=-\infty, \\
0<\lim _{\beta \rightarrow \beta_{0}} l_{2}(\beta)<\infty, & 0<\lim _{\beta \rightarrow \beta_{0}} l_{2}^{\prime}(\beta)<\infty .
\end{array}
$$

Therefore, one can show that the time $\operatorname{map} l_{s}(\beta)=l_{1}(\beta)+l_{2}(\beta)$ is monotone decreasing for $\beta$ near $\beta_{0}\left(\beta>\beta_{0}\right)$. Therefore $l_{s}(\beta)=l$ has a unique solution $\beta=\beta_{s}(l)$ for $\beta \in\left(\beta_{0}, v_{1}\right)$ near $\beta_{0}$ and $l>l_{0}$ for some $l_{0}>0$ (see Figure 9 ). For such a choice of $\beta_{s}(l), x_{s}(l)$ is given by

$$
x_{s}(l)=\int_{v^{*}}^{\beta_{s}(l)} \frac{d v}{\sqrt{2 \int_{v}^{\beta_{s}(l)} g_{+}(s) d s}} .
$$

To construct $V_{a}$, one needs to solve $2 l=l_{1}(\beta)+2 l_{2}(\beta)+l_{3}(\beta)=: 2 l_{a}(\beta)$, where $l_{3}(\beta)$ is the "time" spent between $B$ and $D$ on the phase plane of $v^{\prime \prime}+g_{+}(v)=0$ in Figure 8 and given by

$$
l_{3}(\beta)=\int_{0}^{v^{*}} \frac{d v}{\sqrt{2 \int_{v}^{\beta} g_{+}(s) d s}} .
$$

Therefore, arguing as above, the equation $2 l=2 l_{a}(\beta)$ has a unique solution $\beta=\beta_{a}(l)$ for $\beta \in\left(\beta_{0}, v_{1}\right)$ close to $\beta_{0}$ and $l>l_{0}$. For such choice of $\beta_{a}(l), x_{a}(l)$ is given by

$$
x_{a}(l)=l-2 l_{2}\left(\beta_{a}(l)\right)-l_{3}\left(\beta_{a}(l)\right) .
$$



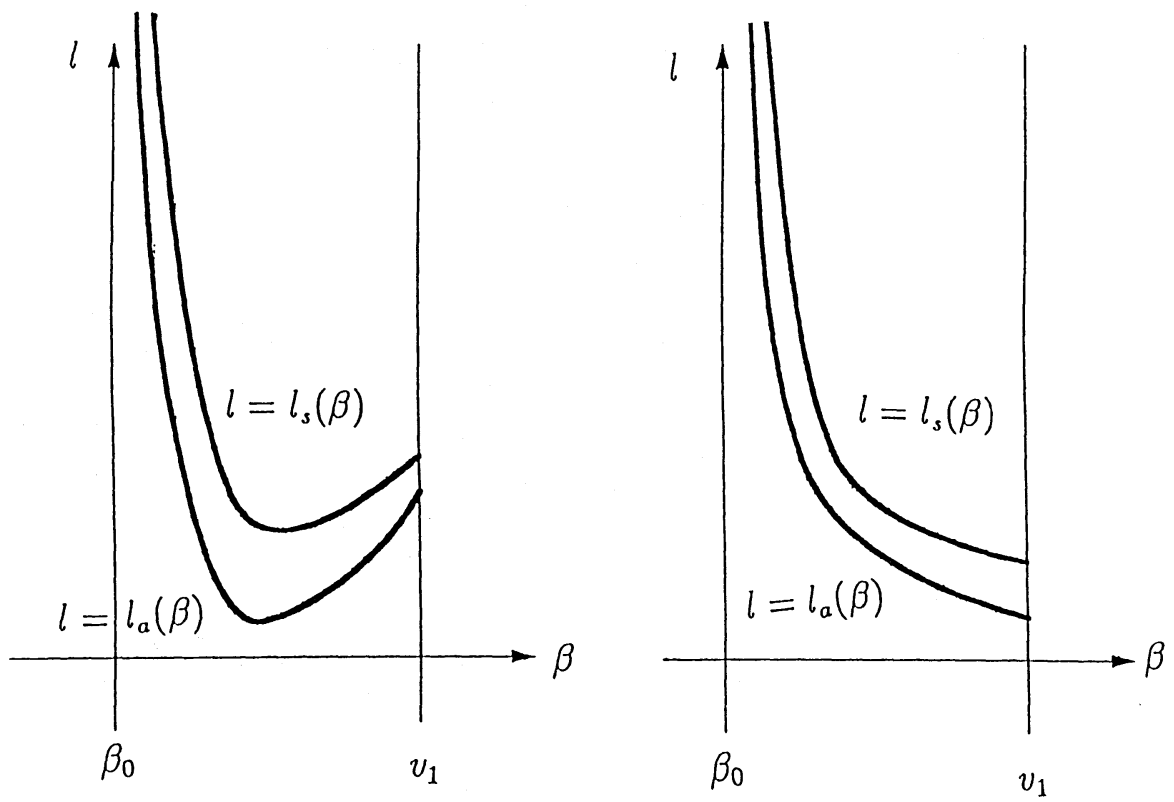

Figure 9. Time maps $l_{s}(\beta)$ and $l_{a}(\beta)$.

2.2. Inner approximation for symmetric solutions. In order to construct the symmetric solutions $\left(u_{s}^{\epsilon}, v_{s}^{\epsilon}\right)$, we work on the half interval $[-l, 0]$. The outer solution $U_{s}$ has a jump discontinuity at $x=-x_{s}(l)$ which can be smoothed out by an inner solution. The inner solution is obtained as follows. Consider the problem

$$
\epsilon^{2} u_{x x}+f\left(u, V_{s}(x)\right)=0 .
$$

By changing variables $u=H\left(V_{s}(x)\right) z+h_{-}\left(V_{s}(x)\right)$ with $H(v)=h_{+}(v)-h_{-}(v)$, and introducing a stretched variable $y=\left(x+x_{s}(l)\right) / \epsilon$, the above equation becomes

$$
H \ddot{z}+2 \epsilon H_{x} \dot{z}+\epsilon^{2}\left(H_{x x} z+\left(h_{-}\right)_{x x}\right)+f\left(H z+h_{-}, V_{s}\left(\epsilon y-x_{s}(l)\right)\right)=0,
$$

where $\dot{z}$ means the derivative of $z$ with respect to $y$ and the functions $H$ and $h_{-}$ are evaluated at $V_{s}\left(\epsilon y-x_{s}(l)\right)$. Substituting the expression $z(y)=z_{0}(y)+\epsilon z_{1}(y)$ in the above and equating the coefficient of each power of $\epsilon$ separately to zero, we have equations for $z_{0}$ and $z_{1}$ :

$$
\begin{gathered}
H\left(v^{*}\right) \ddot{z}_{0}+f\left(H\left(v^{*}\right) z_{0}+h_{-}\left(v^{*}\right), v^{*}\right)=0, \quad y \in(-\infty, \infty), \\
H\left(v^{*}\right) \ddot{z}_{1}+f_{u}(\sharp) H\left(v^{*}\right) z_{1}+\left[f_{u}(\sharp)\left[H^{\prime}\left(v^{*}\right) z_{0}(y)+h_{-}{ }^{\prime}\left(v^{*}\right)\right]+f_{v}(\sharp)\right] V_{s x}\left(v^{*}\right) y \\
+H^{\prime}\left(v^{*}\right) \ddot{z}_{0}(y) V_{s x}\left(v^{*}\right) y+2 H^{\prime}\left(v^{*}\right) \dot{z}_{0}(y) V_{s x}\left(v^{*}\right)=0,
\end{gathered}
$$

where $f_{u}(\sharp)$ and $f_{v}(\sharp)$ are evaluated at $(u, v)=\left(H\left(v^{*}\right) z_{0}+h_{-}\left(v^{*}\right), v^{*}\right)$. It is easy to show that the above equations have solutions satisfying

$$
\lim _{y \rightarrow \infty} z_{0}(y)=1, \quad \lim _{y \rightarrow-\infty} z_{0}(y)=0, \quad \lim _{y \rightarrow \infty} z_{1}( \pm y)=0
$$

with the convergence being at an exponential rate (see Hale and Sakamoto [1988]). Let $Z(y)=z_{0}(y)+\epsilon z_{1}(y)$. We glue the outer solution $U_{s}$ and the inner solution $Z(y)$ 
together. Using smooth cut-off functions $\zeta_{0}(x), \zeta_{1}(x)$ satisfying

$$
\begin{gathered}
\zeta_{0}(x)=1 \text { for }|x| \leq \frac{1}{4 x_{s}(\infty)}, \quad \zeta_{0}(x)=0 \text { for }|x| \geq \frac{1}{2 x_{s}(\infty)}, \quad 0 \leq \zeta_{0}(x) \leq 1, \\
\zeta_{1}(x)=1-\zeta_{0}(x) \text { for } x \geq 0, \quad \zeta_{1}(x)=0 \text { for } x<0
\end{gathered}
$$

we define a tentative approximation for $u$ as

$$
U_{T}^{\epsilon}(x)=H\left(V_{s}(x)\right)\left[Z\left(\frac{x+x_{s}(l)}{\epsilon}\right) \zeta_{0}\left(x+x_{s}(l)\right)+\zeta_{1}\left(x+x_{s}(l)\right)\right]+h_{-}\left(V_{s}(x)\right) .
$$

A tedious computation shows that $\left(U_{T}^{\epsilon}, V_{s}\right)$ satisfies the first equation of (1.3) within the order of $O\left(\epsilon^{2}\right)$ except at the point $x=-x_{s}(l)$. On the other hand, the same pair of functions have only $C^{1}$-regularity. Besides having discontinuous second derivatives at $x=-x_{s}(l)$, the pair $\left(U_{T}^{\epsilon}, V_{s}\right)$ cannot be a good approximation to a solution of (1.3), since we only have

$$
\left|\left(V_{s}\right)_{x x}+g\left(U_{T}^{\epsilon}, V_{s}\right)\right|_{L^{\infty}}=O(1) .
$$

To remedy this defect, we add an appropriate correction to $V_{s}$. Let us define $S_{ \pm}(y)$ by

$$
\begin{array}{r}
S_{-}(y)=-\int_{\left(-l+x_{s}(l)\right) / \epsilon}^{y} \int_{\left(-l+x_{s}(l)\right) / \epsilon}^{t}\left[g\left(U_{T}^{\epsilon}\left(\epsilon s-x_{s}(l)\right), V_{s}\left(\epsilon s-x_{s}(l)\right)\right)\right. \\
\left.-g\left(U_{s}\left(\epsilon s-x_{s}(l)\right), V_{s}\left(\epsilon s-x_{s}(l)\right)\right)\right] d s d t \\
S_{+}(y)=-\int_{y}^{x_{s}(l) / \epsilon} \int_{t}^{x_{s}(l) / \epsilon}\left[g\left(U_{T}^{\epsilon}\left(\epsilon s-x_{s}(l)\right), V_{s}\left(\epsilon s-x_{s}(l)\right)\right)\right. \\
\left.-g\left(U_{s}\left(\epsilon s-x_{s}(l)\right), V_{s}\left(\epsilon s-x_{s}(l)\right)\right)\right] d s d t
\end{array}
$$

and $S(y)$ by

$$
S(y)= \begin{cases}S_{-}(y)-\left[S_{-}(0)+y \dot{S}_{-}(0)\right] \zeta_{0}(\epsilon y), & y \leq 0 \\ S_{+}(y)-\left[S_{+}(0)+y \dot{S}_{+}(0)\right] \zeta_{0}(\epsilon y), & y \geq 0 .\end{cases}
$$

We then define $V_{0}^{\epsilon}$ by

$$
V_{0}^{\epsilon}(x)=V_{s}(x)+\epsilon^{2} S\left(\frac{x+x_{s}(l)}{\epsilon}\right) .
$$

A direct computation similar to that given in Hale and Sakamoto [1988], Sakamoto [1990] shows that $V_{0}^{\epsilon}$ is of $C^{2}$-class, and

$$
\left|V_{0 x x}^{\epsilon}+g\left(U_{T}^{\epsilon}, V_{0}^{\epsilon}\right)\right|_{L^{\infty}}=O(\epsilon) .
$$

By using this, we define $V^{\epsilon}$ and $U^{\epsilon}$ by

$$
V^{\epsilon}(x)=V_{1}^{\epsilon}(x)+V_{0}^{\epsilon}(x)
$$

and

$$
U^{\epsilon}(x)=H\left(V^{\epsilon}(x)\right)\left[Z\left(\frac{x+x_{s}(l)}{\epsilon}\right) \zeta_{0}\left(x+x_{s}(l)\right)+\zeta_{1}\left(x+x_{s}(l)\right)\right]+h_{-}\left(V^{\epsilon}(x)\right),
$$


where $V_{1}^{\epsilon}$ will be chosen so that

$$
V_{x x}^{\epsilon}+g\left(U^{\epsilon}, V^{\epsilon}\right)=0
$$

is satisfied. To see that this is possible, let $U_{0}^{\epsilon}$ be defined by

$$
U_{0}^{\epsilon}(x)=H\left(V_{0}^{\epsilon}(x)\right)\left[Z\left(\frac{x+x_{s}(l)}{\epsilon}\right) \zeta_{0}\left(x+x_{s}(l)\right)+\zeta_{1}\left(x+x_{s}(l)\right)\right]+h_{-}\left(V_{0}^{\epsilon}(x)\right)
$$

and $D_{v} U_{0}^{\epsilon}$ by

$$
D_{v} U_{0}^{\epsilon}(x)=H^{\prime}\left(V_{0}^{\epsilon}(x)\right)\left[Z\left(\frac{x+x_{s}(l)}{\epsilon}\right) \zeta_{0}\left(x+x_{s}(l)\right)+\zeta_{1}\left(x+x_{s}(l)\right)\right]+h_{-}{ }^{\prime}\left(V_{0}^{\epsilon}(x)\right) .
$$

Then equation (2.3) becomes

$$
M_{0}^{\epsilon} V_{1}^{\epsilon}+\left[V_{0 x x}^{\epsilon}+g\left(U_{0}^{\epsilon}, V_{0}^{\epsilon}\right)\right]+G^{\epsilon}\left(V_{1}^{\epsilon}\right)=0,
$$

where

$$
\begin{aligned}
M_{0}^{\epsilon} V_{1}^{\epsilon} & =V_{1 x x}^{\epsilon}+\left[g_{u}\left(U_{0}^{\epsilon}, V_{0}^{\epsilon}\right) D_{v} U_{0}^{\epsilon}+g_{v}\left(U_{0}^{\epsilon}, V_{0}^{\epsilon}\right)\right] V_{1}^{\epsilon}, \\
G^{\epsilon}\left(V_{1}^{\epsilon}\right) & =g\left(U^{\epsilon}, V^{\epsilon}\right)-g\left(U_{0}^{\epsilon}, V_{0}^{\epsilon}\right)-\left[g_{u}\left(U_{0}^{\epsilon}, V_{0}^{\epsilon}\right) D_{v} U_{0}^{\epsilon}+g_{v}\left(U_{0}^{\epsilon}, V_{0}^{\epsilon}\right)\right] V_{1}^{\epsilon} \\
& =O\left(\left|V_{1}^{\epsilon}\right|^{2}\right) .
\end{aligned}
$$

Lemma 1. The operator $M_{0}^{\epsilon}: H_{D N}^{2} \rightarrow H^{0}$ has an inverse whose norm is bounded uniformly in $\epsilon \in\left(0, \epsilon_{0}\right], l \in\left(l_{0}, \infty\right)$.

For the proof of this lemma, see Lemma 3.3 of Sakamoto [1990].

Lemma 1 implies that the equation (2.4) has a unique solution $V_{1}^{\epsilon}$ with the property $\left\|V_{1}^{\epsilon}\right\|_{H^{2}}=O(\epsilon)$. We choose $\left(U^{\epsilon}, V^{\epsilon}\right)$ to be our approximate solution to the problem (1.3) and seek a true solution nearby. It turns out to be convenient to change variables in (1.3) for the subsequent analyses. By introducing a new set of variables $(\underline{u}, \underline{v})$ via

$$
u=U^{\epsilon}+\underline{u}+D_{v} U^{\epsilon} \underline{v}, \quad v=V^{\epsilon}+\underline{v},
$$

where

$$
D_{v} U^{\epsilon}(x)=H^{\prime}\left(V^{\epsilon}(x)\right)\left[Z\left(\frac{x+x_{s}(l)}{\epsilon}\right) \zeta_{0}\left(x+x_{s}(l)\right)+\zeta_{1}\left(x+x_{s}(l)\right)\right]+h_{-}^{\prime}\left(V^{\epsilon}(x)\right),
$$

the equation (1.3) for the new variables $\underline{u}, \underline{v}$ becomes (after dropping the underlines)

$$
\begin{aligned}
& L^{\epsilon} u+N^{\epsilon} v+R_{1}^{\epsilon}+F_{1}^{\epsilon}(u, v)=0, \\
& M^{\epsilon} v+g_{u}^{\epsilon} v+R_{2}^{\epsilon}+F_{2}^{\epsilon}(u, v)=0,
\end{aligned}
$$

where $L^{\epsilon}: H_{D N}^{2} \rightarrow H^{0}, N^{\epsilon}: H_{D N}^{2} \rightarrow H^{0}$, and $M^{\epsilon}: H_{D N}^{2} \rightarrow H^{0}$ are second-order differential operators defined by

$$
\begin{aligned}
L^{\epsilon} u & =\epsilon^{2} u_{x x}+f_{u}\left(U^{\epsilon}, V^{\epsilon}\right) u \\
N^{\epsilon} v & =L^{\epsilon}\left(D_{v} U^{\epsilon} v\right)+f_{v}\left(U^{\epsilon}, V^{\epsilon}\right) v \\
M^{\epsilon} v & =v_{x x}+\left[g_{u}\left(U^{\epsilon}, V^{\epsilon}\right) D_{v} U^{\epsilon}+g_{v}\left(U^{\epsilon}, V^{\epsilon}\right)\right] v
\end{aligned}
$$

and $g_{u}^{\epsilon}=g_{u}\left(U^{\epsilon}, V^{\epsilon}\right)$. The terms $R_{1}^{\epsilon}, R_{2}^{\epsilon}$ are residues:

$$
R_{1}^{\epsilon}=\epsilon^{2} U_{x x}^{\epsilon}+f\left(U^{\epsilon}, V^{\epsilon}\right), \quad R_{2}^{\epsilon}=V_{x x}^{\epsilon}+g\left(U^{\epsilon}, V^{\epsilon}\right) \equiv 0 .
$$


A computation similar to that given in Hale and Sakamoto [1988] shows that $\left\|R_{1}^{\epsilon}\right\|_{H^{0}}=$ $O\left(\epsilon^{2}\right)$. The terms $F_{1}^{\epsilon}, F_{2}^{\epsilon}$ are higher-order ones in $(u, v)$, and given by

$$
\begin{aligned}
F_{1}^{\epsilon}(u, v)= & f\left(U^{\epsilon}+u+D_{v} U^{\epsilon} v, V^{\epsilon}+v\right)-f\left(U^{\epsilon}, V^{\epsilon}\right)-f_{u}\left(U^{\epsilon}, V^{\epsilon}\right) u \\
& -\left[f_{u}\left(U^{\epsilon}, V^{\epsilon}\right) D_{v} U^{\epsilon}+f_{v}\left(U^{\epsilon}, V^{\epsilon}\right)\right] v \\
F_{2}^{\epsilon}(u, v)= & g\left(U^{\epsilon}+u+D_{v} U^{\epsilon} v, V^{\epsilon}+v\right)-g\left(U^{\epsilon}, V^{\epsilon}\right)-g_{u}\left(U^{\epsilon}, V^{\epsilon}\right) u \\
& -\left[g_{u}\left(U^{\epsilon}, V^{\epsilon}\right) D_{v} U^{\epsilon}+g_{v}\left(U^{\epsilon}, V^{\epsilon}\right)\right] v .
\end{aligned}
$$

We will show that the problem $(2.6)$ is solvable near $(u, v)=(0,0)$. For this purpose, some properties of the linear operators $L^{\epsilon}, M^{\epsilon}, N^{\epsilon}$ will be studied in the next section.

2.3. Inner approximation for antisymmetric solutions. In order to construct antisymmetric solutions $\left(u_{a}^{\epsilon}, v_{a}^{\epsilon}\right)$, we follow the procedure of the previous subsection. The outer solution $U_{a}$ has a jump discontinuity at $x=x_{a}(l)$ and fails to satisfy the boundary condition at $x=l$. The jump discontinuity can be smoothed out in the same manner as in the previous subsection, while a boundary-layer correction will make the boundary condition satisfied at $x=l$.

Let us start with the boundary-layer correction. Consider the equation

$$
\epsilon^{2} u_{x x}+f\left(u, V_{a}(x)\right)=0 .
$$

Using a stretched variable $y=(x-l) / \epsilon$, and substituting $u=u_{0}^{b}+\epsilon u_{1}^{b}$, we obtain the following problems for $u_{0}^{b}$ and $u_{1}^{b}$ :

$$
\begin{gathered}
\ddot{u}_{0}^{b}+f\left(u_{0}^{b}, V_{a}(l)\right)=0, \quad u_{0}^{b}(0)=0, \quad u_{0}^{b}(-\infty)=U_{a}(l), \\
\ddot{u}_{1}^{b}+f_{u}\left(u_{0}^{b}, V_{a}(l)\right) u_{1}^{b}+f_{v}\left(u_{0}^{b}, V_{a}(l)\right) V_{a x}(l) y=0, \\
u_{1}^{b}(0)=0, \quad u_{1}^{b}(-\infty)=0 .
\end{gathered}
$$

These problems have a unique solution, and $u_{0}^{b}(y)-U_{a}(l)$ and $u_{1}^{b}(y)$ decay exponentially at $y=-\infty$ (see Fife [1976]). We now let

$$
U_{b}^{\epsilon}(y)=u_{0}^{b}(y)+\epsilon u_{1}^{b}(y) .
$$

We now turn to an internal-layer correction. This is almost identical to the previous subsection. Changing variables from $u$ to $z$ via $u=H\left(V_{a}(x)\right) z+h_{-}\left(V_{a}(x)\right)$ and introducing a stretched variable $y=\left(x-x_{a}(l)\right) / \epsilon$, one obtains an internal correction

$$
Z^{\epsilon}(y)=z_{0}(y)+\epsilon z_{1}(y)
$$

where

$$
\lim _{y \rightarrow \infty} z_{0}(y)=1, \quad \lim _{y \rightarrow-\infty} z_{0}(y)=0, \quad \lim _{y \rightarrow \infty} z_{1}( \pm y)=0
$$

with the convergence being at an exponential rate. In order to glue $U_{a}, U_{b}^{\epsilon}$, and $Z^{\epsilon}$ together, we choose smooth cut-off functions $\zeta_{0}, \zeta_{1}$, satisfying

$$
\zeta_{0}(x)=1, \quad \text { for }|x| \leq \frac{1}{4 l_{\infty}}, \quad \zeta_{0}(x)=0, \quad \text { for }|x| \geq \frac{1}{2 l_{\infty}}, \quad 0 \leq \zeta_{0}(x) \leq 1,
$$

where $l_{\infty}=\lim _{l \rightarrow \infty}\left(l-x_{a}(l)\right)$, and

$$
\zeta_{1}(x)=1-\zeta_{0}(x), \quad \text { for } x \geq 0, \quad \zeta_{1}(x)=0 \quad \text { for } x<0 .
$$


By using these, we define a tentative approximation for $u$ as

$$
\begin{aligned}
U_{T}^{\epsilon}(x)=H & \left(V_{a}(x)\right)\left[Z\left(\frac{x-x_{a}(l)}{\epsilon}\right) \zeta_{0}\left(x-x_{a}(l)\right)+\zeta_{1}\left(x-x_{a}(l)\right)\right] \\
+ & {\left[U_{b}^{\epsilon}\left(\frac{x-l}{\epsilon}\right)-U_{a}(l)\right] \zeta_{0}(x-l)+h_{-}\left(V_{a}(x)\right) . }
\end{aligned}
$$

We define $S_{ \pm}(y)$, and $S_{0}(y)$ by

$$
\begin{gathered}
S_{-}(y)=-\int_{\left(-l-x_{a}(l)\right) / \epsilon}^{y} \int_{\left(-l-x_{a}(l)\right) / \epsilon}^{t}\left[g\left(U_{T}^{\epsilon}\left(\epsilon s+x_{a}(l)\right), V_{a}\left(\epsilon s+x_{a}(l)\right)\right)\right. \\
\left.-g\left(U_{a}\left(\epsilon s+x_{a}(l)\right), V_{a}\left(\epsilon s+x_{a}(l)\right)\right)\right] d s d t \\
S_{+}(y)=-\int_{y}^{\left(l-x_{a}(l)\right) / 2 \epsilon} \int_{t}^{\left(l-x_{a}(l)\right) / 2 \epsilon} \begin{array}{c}
{\left[g\left(U_{T}^{\epsilon}\left(\epsilon s+x_{a}(l)\right), V_{a}\left(\epsilon s+x_{a}(l)\right)\right)\right.} \\
\left.-g\left(U_{a}\left(\epsilon s+x_{a}(l)\right), V_{a}\left(\epsilon s+x_{a}(l)\right)\right)\right] d s d t
\end{array} \\
S_{0}(y)=-\int_{\left(-l+x_{a}(l)\right) / 2 \epsilon}^{y} \int_{\left(-l+x_{a}(l)\right) / 2 \epsilon}\left[g\left(U_{T}^{\epsilon}(\epsilon s+l), V_{a}(\epsilon s+l)\right)\right. \\
\left.-g\left(U_{a}(\epsilon s+l), V_{a}(\epsilon s+l)\right)\right] d s d t .
\end{gathered}
$$

Note that the upper limits in the definition of $S_{+}(y)$ and the lower limits in the definition of $S_{0}(y)$ in the above are specified as they appear to make $S^{\epsilon}(x)$ (to be defined below) $C^{2}$-matched at $x=\left(l+x_{a}(l)\right) / 2$. We also define $S(y), S_{b}(y)$ by

$$
\begin{gathered}
S(y)= \begin{cases}S_{-}(y)-\left[S_{-}(0)+y \dot{S}_{-}(0)\right] \zeta_{0}(\epsilon y), & y \leq 0, \\
S_{+}(y)-\left[S_{+}(0)+y \dot{S}_{+}(0)\right] \zeta_{0}(\epsilon y), & y \geq 0,\end{cases} \\
S_{b}(y)=S_{0}(y)-S_{0}(0) \zeta_{0}(\epsilon y), \quad y \leq 0 .
\end{gathered}
$$

By using these, $S^{\epsilon}$ is defined by

$$
S^{\epsilon}(x)=S\left(\frac{x-x_{a}(l)}{\epsilon}\right)+S_{b}\left(\frac{x-l}{\epsilon}\right),
$$

and $V_{0 a}^{\epsilon}$ is defined by

$$
V_{0 a}^{\epsilon}(x)=V_{a}(x)+\epsilon^{2} S^{\epsilon}(x) .
$$

One should notice that $V_{0 a}^{\epsilon}$ is of $C^{2}$-class, and that

$$
\left|\left(V_{0 a}^{\epsilon}\right)_{x x}+g\left(U_{T}^{\epsilon}, V_{0 a}^{\epsilon}\right)\right|_{L^{\infty}}=O(\epsilon) .
$$

We finally define $V_{a}^{\epsilon}$ and $U_{a}^{\epsilon}$ by

$$
V_{a}^{\epsilon}(x)=V_{1 a}^{\epsilon}(x)+V_{0 a}^{\epsilon}(x)
$$

and

$$
\begin{aligned}
U_{a}^{\epsilon}(x)= & H\left(V_{a}^{\epsilon}(x)\right)\left[Z\left(\frac{x-x_{a}(l)}{\epsilon}\right) \zeta_{0}\left(x-x_{a}(l)\right)+\zeta_{1}\left(x-x_{a}(l)\right)\right] \\
& +\left[U_{b}^{\epsilon}\left(\frac{x-l}{\epsilon}\right)-U_{a}(l)\right] \zeta_{0}(x-l)+h_{-}\left(V_{a}^{\epsilon}(x)\right),
\end{aligned}
$$

where $V_{1 a}^{\epsilon}$ will be chosen so that

$$
\left(V_{a}^{\epsilon}\right)_{x x}+g\left(U_{a}^{\epsilon}, V_{a}^{\epsilon}\right)=0
$$


is satisfied. The remaining part follows from the same line of arguments as the previous subsection, and we arrive at

$$
\begin{aligned}
& L_{a}^{\epsilon} u+N_{a}^{\epsilon} v+R_{1 a}^{\epsilon}+F_{1 a}^{\epsilon}(u, v)=0, \\
& M_{a}^{\epsilon} v+g_{u}^{\epsilon} v+R_{2 a}^{\epsilon}+F_{2 a}^{\epsilon}(u, v)=0,
\end{aligned}
$$

where $L_{a}^{\epsilon}: H_{D D}^{2}[-l, l] \rightarrow H^{0}[-l, l], N_{a}^{\epsilon}: H_{D D}^{2}[-l, l] \rightarrow H^{0}[-l, l]$, and $M_{a}^{\epsilon}: H_{D D}^{2}[-l, l]$ $\rightarrow H^{0}[-l, l]$ are the second-order differential operators defined by

$$
\begin{aligned}
L_{a}^{\epsilon} u & =\epsilon^{2} u_{x x}+f_{u}\left(U_{a}^{\epsilon}, V_{a}^{\epsilon}\right) u \\
N_{a}^{\epsilon} v & =L_{a}^{\epsilon}\left(D_{v} U_{a}^{\epsilon} v\right)+f_{v}\left(U_{a}^{\epsilon}, V_{a}^{\epsilon}\right) v \\
M_{a}^{\epsilon} v & =v_{x x}+\left[g_{u}\left(U_{a}^{\epsilon}, V_{a}^{\epsilon}\right) D_{v} U_{a}^{\epsilon}+g_{v}\left(U_{a}^{\epsilon}, V_{a}^{\epsilon}\right)\right] v
\end{aligned}
$$

and $g_{u}^{\epsilon}=g_{u}\left(U_{a}^{\epsilon}, V_{a}^{\epsilon}\right)$. The terms $R_{1 a}^{\epsilon}, R_{2 a}^{\epsilon}$ are residues:

$$
R_{1 a}^{\epsilon}=\epsilon^{2} U_{a x x}^{\epsilon}+f\left(U_{a}^{\epsilon}, V_{a}^{\epsilon}\right), \quad R_{2 a}^{\epsilon}=V_{a x x}^{\epsilon}+g\left(U_{a}^{\epsilon}, V_{a}^{\epsilon}\right) \equiv 0 .
$$

A computation similar to that given in Hale and Sakamoto [1988] shows $\left\|R_{1 a}^{\epsilon}\right\|_{H^{\circ}[-l, l]}=$ $O\left(\epsilon^{2}\right) . F_{1 a}^{\epsilon}, F_{2 a}^{\epsilon}$ are higher-order terms given by formulas similar to those of $F_{1}^{\epsilon}, F_{2}^{\epsilon}$.

\section{Linear analysis}

In the previous section, the existence of transition-layer solutions was reduced to showing the solvability of the equations (2.6) or (2.11) on appropriate function spaces. The key in this setting is detailed information on the linear operators $L^{\epsilon}$, etc.

3.1. Analysis for symmetric solutions. In this subsection, we consider the operators $L^{\epsilon}, M^{\epsilon}$, and $N^{\epsilon}$ with the two sets of boundary conditions

and

$$
\text { (DN) } \quad u(-l)=0=u_{x}(0), \quad v(-l)=0=v_{x}(0)
$$

$$
\text { (DD) } \quad u(-l)=0=u(0), \quad v(-l)=0=v(0) \text {. }
$$

All the results in this subsection are valid for both of these boundary conditions unless stated otherwise. We refer the reader to Sakamoto [1990] for proofs of Lemmas 2, 3, 4 , and 6 .

Lemma 2. Let $\left\{\phi_{n}^{\epsilon}, \lambda_{n}^{\epsilon}\right\}_{n=0}^{\infty}$ be a system of complete orthornomal eigenpairs of the operator $L^{\epsilon}$.

(i) There exist $\delta_{0}>0, \epsilon_{0}>0$ such that $\lambda_{1}^{\epsilon} \leq-\delta_{0}$, for $\epsilon \in\left(0, \epsilon_{0}\right)$.

(ii) $\lim _{\epsilon \rightarrow 0} \lambda_{0}^{\epsilon}=0$ uniformly in $l \in\left(l_{0}, \infty\right)$.

(iii) There are constants $k>0, \beta>0$ such that

$$
\left|\phi_{0}^{\epsilon}(x)\right| \leq k\left|\phi_{0}^{\epsilon}\left(-x_{s}(l)\right)\right| \exp \left(\frac{-\beta\left|x+x_{s}(l)\right|}{\epsilon}\right) .
$$

(iv) If the eigenfunction $\phi_{0}^{\epsilon}$ is normalized so that $\phi_{0}^{\epsilon}(0)>0$, then we have

$$
\sqrt{\epsilon} \phi_{0}^{\epsilon}\left(\epsilon y-x_{s}(l)\right) \rightarrow K_{*} H\left(v^{*}\right) \dot{z}_{0}(y), \quad \text { as } \quad \epsilon \rightarrow 0 \quad \text { in } C_{l o c}^{2}(-\infty, \infty)
$$

uniformly in $l \in\left(l_{0}, \infty\right)$, where $K_{*}^{-1}$ is

$$
K_{*}^{-1}=\sqrt{\int_{-\infty}^{\infty}\left|H\left(v^{*}\right) \dot{z}_{0}(y)\right|^{2} d y}
$$


(v) The following limit exists.

$$
\lim _{\epsilon \rightarrow 0} \frac{\lambda_{0}^{\epsilon}}{\epsilon}=-K_{*}^{2} J^{\prime}\left(v^{*}\right) V_{s x}\left(-x_{s}(l)\right)=: \lambda_{0}^{*} .
$$

Lemma 3. There exists a constant $\delta_{1}>0$ such that the principal eigenvalue $\mu_{0}^{\epsilon}$ of $M^{\epsilon}: H_{D N}^{2}\left(\right.$ or $\left.H_{D D}^{2}\right) \rightarrow H^{0}$ satisfies $\mu_{0}^{\epsilon}<-\delta_{1}$ for $\epsilon \in\left(0, \epsilon_{0}\right]$ and $l \in\left(l_{0}, \infty\right)$.

In order to solve the eigenvalue problem (3.1) below, we need some estimates on operators obtained from $M^{\epsilon}$ and $N^{\epsilon}$.

Lemma 4. The following statements are valid as $\epsilon \rightarrow 0$, uniformly in $l \in\left(l_{0}, \infty\right)$, and $\Re \mu>-\delta_{1}$.

(i) $\left\|N^{\epsilon}\left(M^{\epsilon}-\mu\right)^{-1}\right\|_{H^{0} \rightarrow H^{0}}=O(\sqrt{\epsilon})$;

(ii) $\left\|\left(M^{\epsilon}-\mu\right)^{-1} g_{u}^{\epsilon} \phi_{0}^{\epsilon}\right\|_{L^{\infty}}=O(\sqrt{\epsilon})$;

(iii) $\left\|N^{\epsilon}\left(M^{\epsilon}-\mu\right)^{-1} g_{u}^{\epsilon} \phi_{0}^{\epsilon}\right\|_{H^{0}}=O(\epsilon)$;

(iv) $\left\|\left(M^{\epsilon}-\mu\right)^{-1} N_{*}^{\epsilon}\right\|_{H^{2} \rightarrow H^{2}}=O(\sqrt{\epsilon})$, where $N_{*}^{\epsilon}$ is the adjoint of $N^{\epsilon}$.

We consider the following eigenvalue problem.

$$
\left(\begin{array}{ll}
L^{\epsilon} & N^{\epsilon} \\
g_{u}^{\epsilon} & M^{\epsilon}
\end{array}\right)\left(\begin{array}{c}
w \\
z
\end{array}\right)=\rho\left(\begin{array}{c}
w \\
z
\end{array}\right), \quad w, z \in H_{D N}^{2}
$$

For this problem, we have

Lemma 5. Let $\rho_{0}=\min \left(\delta_{0}, \delta_{1}\right)$. The eigenvalue problem (3.1) has a unique, simple, real eigenvalue $\rho^{\epsilon}$ in the region $\Re \rho>-\rho_{0}$. Moreover, $\rho^{\epsilon}$ has the asymptotic expression

$$
\rho^{\epsilon}=\epsilon \rho^{*}+o(\epsilon), \text { where } \rho^{*}<0 .
$$

Proof. Lemma 2 implies that the second equation of the eigenvalue problem (3.1) can be solved in $z$ as

$$
z=-\left(M^{\epsilon}-\rho\right)^{-1} g_{u}^{\epsilon} w=-\left(M^{\epsilon}-\rho\right)^{-1} g_{u}^{\epsilon}\left(\alpha \phi_{0}^{\epsilon}+w_{1}\right), \quad \text { where }\left\langle w_{1}, \phi_{0}^{\epsilon}\right\rangle=0
$$

for $\Re \rho>-\mu_{0}$. Substituting this into the first equation, one obtains

$$
\begin{gathered}
\alpha\left(\lambda_{0}^{\epsilon}-\rho\right)-\left\langle\left(M^{\epsilon}-\rho\right)^{-1} g_{u}^{\epsilon}\left(\alpha \phi_{0}^{\epsilon}+w_{1}\right), N_{*}^{\epsilon} \phi_{0}^{\epsilon}\right\rangle=0 \\
\left(L^{\epsilon}-\rho\right) w_{1}-Q N^{\epsilon}\left(M^{\epsilon}-\rho\right)^{-1} g_{u}^{\epsilon} w_{1}=\alpha Q N^{\epsilon}\left(M^{\epsilon}-\rho\right)^{-1} g_{u}^{\epsilon} \phi_{0}^{\epsilon},
\end{gathered}
$$

where $Q$ is the orthogonal projection onto the complement of the span of $\phi_{0}^{\epsilon}$ in $H^{0}$. By Lemma 3(iii), the second equation in the above can be solved in $w_{1}$ as

$$
w_{1}=\alpha K^{\epsilon, \rho} \phi_{0}^{\epsilon}, \quad K^{\epsilon, \rho} u=\sum_{n \geq 0}\left[\left(L^{\epsilon}-\rho\right)^{-1} Q N^{\epsilon}\left(M^{\epsilon}-\rho\right)^{-1} g_{u}^{\epsilon}\right]^{n+1} u .
$$

Therefore, $\rho$ in the region $\Re \rho>-\rho_{0}$ is an eigenvalue of the problem (3.1) if and only if

$$
\left(\lambda_{0}^{\epsilon}-\rho\right)-\left\langle\left(M^{\epsilon}-\rho\right)^{-1} g_{u}^{\epsilon}\left(\phi_{0}^{\epsilon}+K^{\epsilon, \rho} \phi_{0}^{\epsilon}\right), N_{*}^{\epsilon} \phi_{0}^{\epsilon}\right\rangle=0
$$

is satisfied. The operator $K^{\epsilon, \rho}$ satisfies the following

Lemma 6. The statements (a), (b), and (c) below are valid uniformly in $\epsilon \in\left(0, \epsilon_{0}\right]$, $l \in\left(l_{0}, \infty\right)$, and $\Re \rho>-\rho_{0}$.

(a) $K^{\epsilon, \rho}: H^{0} \rightarrow H_{D N, \epsilon}^{2}\left(\right.$ or $\left.H_{D D, \epsilon}^{2}\right)$ is bounded.

(b) $\left\|K^{\epsilon, \rho} \phi_{0}^{\epsilon}\right\|_{H^{0}}=O(\epsilon)$. 
(c) $\quad\left\|\left(M^{\epsilon}-\rho\right)^{-1} g_{u}^{\epsilon} K^{\epsilon, \rho} \phi_{0}^{\epsilon}\right\|_{H^{2}}=O(\epsilon)$.

Therefore $\rho=O(\epsilon)$ follows, so that one can put $\rho=\epsilon \rho^{*}+o(\epsilon)$. We now compute $\rho^{*}$

$$
\lambda_{0}^{*}-\rho^{*}=\lim _{\epsilon \rightarrow 0} \frac{1}{\epsilon}\left\langle\left(M^{\epsilon}-\left(\epsilon \rho^{*}+o(\epsilon)\right)\right)^{-1} g_{u}^{\epsilon} \phi_{0}^{\epsilon}, f_{v}^{\epsilon} \phi_{0}^{\epsilon}\right\rangle .
$$

The limit in the last equation can be rewritten as

$$
\begin{aligned}
\lim _{\epsilon \rightarrow 0} \frac{1}{\epsilon}\left\langle\left(M^{\epsilon}-\left(\epsilon \rho^{*}+o(\epsilon)\right)\right)^{-1} g_{u}^{\epsilon} \phi_{0}^{\epsilon}, f_{v}^{\epsilon} \phi_{0}^{\epsilon}\right\rangle & =\lim _{\epsilon \rightarrow 0}\left\langle\left(M^{\epsilon}\right)^{-1} g_{u}^{\epsilon} \phi_{0}^{\epsilon} / \sqrt{\epsilon}, f_{v}^{\epsilon} \phi_{0}^{\epsilon} / \sqrt{\epsilon}\right\rangle \\
& =\lim _{\epsilon \rightarrow 0}\left\langle z^{\epsilon}, f_{v}^{\epsilon} \phi_{0}^{\epsilon} / \sqrt{\epsilon}\right\rangle
\end{aligned}
$$

where

$$
z^{\epsilon}=\left(M^{\epsilon}\right)^{-1} g_{u}^{\epsilon} \phi_{0}^{\epsilon} / \sqrt{\epsilon}
$$

In order to evaluate the last limit, the following lemma is essential. One should keep in mind that $z^{\epsilon} \in H_{D N}^{2}$ here.

Lemma 7. (a) For each $\psi \in H^{1}$,

$$
\lim _{\epsilon \rightarrow 0}\left\langle\psi, f_{v}^{\epsilon} \phi_{0}^{\epsilon} / \sqrt{\epsilon}\right\rangle=K_{*} J^{\prime}\left(v^{*}\right) \psi\left(-x_{s}(l)\right) .
$$

(b) As $\epsilon \rightarrow 0$, the function $z^{\epsilon}$ has a limit $z^{*}$ in $H^{1}$ which satisfies $-z_{x}^{*}(-l) \psi(-l)-\left\langle z_{x}^{*}, \psi_{x}\right\rangle+\left\langle B^{*} z^{*}, \psi\right\rangle=K_{*}\left[g_{+}\left(v^{*}\right)-g_{-}\left(v^{*}\right)\right] \psi\left(-x_{s}(l)\right), \quad$ for $\psi \in H^{1}$, where $B^{*}=g_{*}{ }^{\prime}\left(V_{s}(x)\right)$.

For the proof of this lemma, see Nishiura and Fujii [1987].

From this lemma, we know

$$
\lim _{\epsilon \rightarrow 0}\left\langle z^{\epsilon}, f_{v}^{\epsilon} \phi_{0}^{\epsilon} / \sqrt{\epsilon}\right\rangle=K_{*} J^{\prime}\left(v^{*}\right) z^{*}\left(-x_{s}(l)\right) .
$$

If one takes $\psi=V_{s x}$ as a test function in Lemma $7(\mathrm{~b})$, and uses the fact that $V_{s x x x}+$ $B^{*} V_{s x}=0$ other than the point $x=-x_{s}(l)$, then one finds, by using integration by parts, that

$$
z^{*}\left(-x_{s}(l)\right)=-K_{*} V_{s x}\left(-x_{s}(l)\right)-\frac{z^{*}(0) V_{s x x}(0)+z_{x}^{*}(-l) V_{s x}(-l)}{g_{+}\left(v^{*}\right)-g_{-}\left(v^{*}\right)} .
$$

Therefore, recalling $\lambda_{0}^{*}=-K_{*}^{2} J^{\prime}\left(v^{*}\right) V_{s x}\left(-x_{s}(l)\right)$, we have

$$
\rho^{*}=K_{*} J^{\prime}\left(v^{*}\right) \frac{z_{x}^{*}(-l) V_{s x}(-l)+z^{*}(0) V_{s x x}(0)}{g_{+}\left(v^{*}\right)-g_{-}\left(v^{*}\right)} .
$$

Taking $\psi=1$ as a test function in (b) of Lemma 7 , one obtains,

$$
-z_{x}^{*}(-l)+\int_{-l}^{1} B^{*}(x) z^{*}(x) d x=K_{*}\left[g_{+}\left(v^{*}\right)-g_{-}\left(v^{*}\right)\right]>0 .
$$

Since $z^{*}$ satisfies $z_{x x}^{*}+B^{*}(x) z^{*}=0$ for $x \neq-x_{s}(l)$, if $z_{x}^{*}(-l)>0$, then $z^{*}(x)>0$ is true for $x \neq-l$, which contradicts the relationship in the above. Hence, $z^{*}(0)<0$ and $z_{x}^{*}(-l)<0$ follow. Also, an easy comparison argument implies

$$
\left|z_{x}^{*}(-l)\right| \leq \frac{\alpha \cosh \left(\beta x_{s}(l)\right)}{\sinh \left(\alpha\left(l-x_{s}(l)\right)\right)}\left|z^{*}(0)\right|
$$


where $\alpha$ and $\beta$ are constants satisfying

$$
0<\alpha<\min _{-l \leq x \leq 0}\left|B^{*}(x)\right|, \quad \beta>\max _{-l \leq x \leq 0}\left|B^{*}(x)\right| .
$$

Therefore $\left|z_{x}^{*}(-l)\right| /\left|z^{*}(0)\right| \rightarrow 0$ as $l \rightarrow \infty$. Moreover, $0<V_{s x}(-l) \rightarrow 0$ as $l \rightarrow \infty$ and $V_{s x x}(0)<0$ is bounded away from zero as $l \rightarrow \infty$. Therefore for large $l$, say $l>l_{0}, \rho^{*}$ is negative (see Figure 10). The simplicity of the eigenvalue in Lemma 5 can be proved

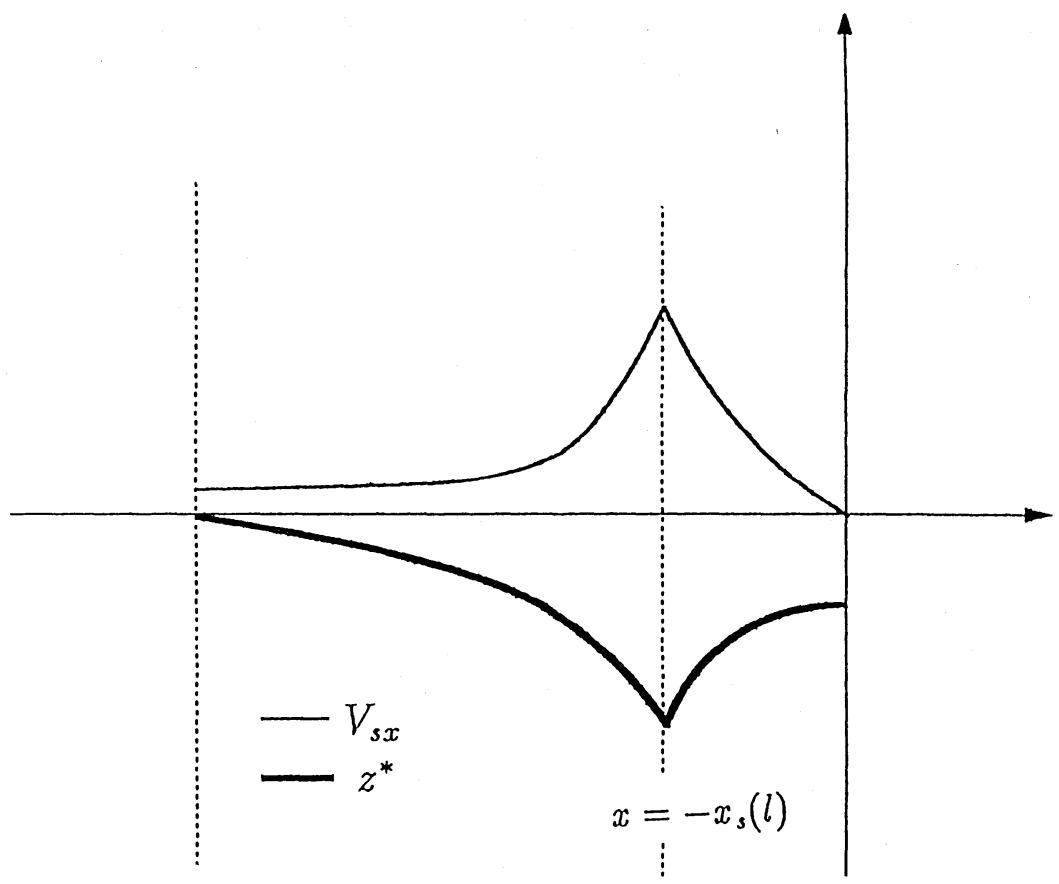

Figure 10. Profiles of $z^{*}$ and $V_{s x}$.

in a manner similar to Sakamoto [1990] by using Lemma 4 (iv). This completes the proof of Lemma 5. In the next section, we will show the solvability of the equation (2.6) via the method of Liapunov-Schmidt.

3.2. Analysis for antisymmetric solutions. Since the analysis for antisymmetric solutions is almost identical to that of symmetric solutions, we will not repeat the details. We will also use frequently the same symbols as in the previous subsection to represent objects which have an apparent counterpart there.

We consider the following eigenvalue problem

$$
\left(\begin{array}{cc}
L_{a}^{\epsilon} & N_{a}^{\epsilon} \\
g_{u}^{\epsilon} & M_{a}^{\epsilon}
\end{array}\right)\left(\begin{array}{c}
w \\
z
\end{array}\right)=\rho\left(\begin{array}{c}
w \\
z
\end{array}\right), \quad x \in(-l, l)
$$

with the boundary condition

$$
w( \pm l)=0=z( \pm l)
$$

In this subsection, we prove the following. 
Lemma 8. There exists a $\rho_{0}>0$ such that the eigenvalue problem (3.2) has a unique, simple, real eigenvalue $\rho^{\epsilon}$ in the region $\Re \rho>-\rho_{0}$. Moreover, $\rho^{\epsilon}$ has the asymptotic expression

$$
\rho^{\epsilon}=\epsilon \hat{\rho}+o(\epsilon), \quad \text { where } \hat{\rho}<0
$$

The operators $L_{a}^{\epsilon}, M_{a}^{\epsilon}$, and $N_{a}^{\epsilon}$ have properties similar to those for $L^{\epsilon}$, etc., and the operator $L_{a}^{\epsilon}$ has one small eigenvalue (of order $O(\epsilon)$ ). The boundary layer in the antisymmetric solutions does not contribute to introduce a small eigenvalue of $L_{a}^{\epsilon}$. Following the same line of arguments as in Section 3.1, one arrives at

$$
\lambda_{0}^{*}-\hat{\rho}=\lim _{\epsilon \rightarrow 0} \frac{1}{\epsilon}\left\langle\left(M_{a}^{\epsilon}-(\epsilon \hat{\rho}+o(\epsilon))\right)^{-1} g_{u}^{\epsilon} \phi_{0}^{\epsilon}, f_{v}^{\epsilon} \phi_{0}^{\epsilon}\right\rangle,
$$

where $\lambda_{0}^{*}=-K_{*}^{2} J^{\prime}\left(v^{*}\right) V_{a}\left(x_{a}(l)\right)$. The limit in the last equation can be rewritten as

$$
\begin{aligned}
\lim _{\epsilon \rightarrow 0} \frac{1}{\epsilon}\left\langle\left(M_{a}^{\epsilon}-(\epsilon \hat{\rho}+o(\epsilon))\right)^{-1} g_{u}^{\epsilon} \phi_{0}^{\epsilon}, f_{v}^{\epsilon} \phi_{0}^{\epsilon}\right\rangle & =\lim _{\epsilon \rightarrow 0}\left\langle\left(M_{a}^{\epsilon}\right)^{-1} g_{u}^{\epsilon} \phi_{0}^{\epsilon} / \sqrt{\epsilon}, f_{v}^{\epsilon} \phi_{0}^{\epsilon} / \sqrt{\epsilon}\right\rangle \\
& =: \lim _{\epsilon \rightarrow 0}\left\langle z^{\epsilon}, f_{v}^{\epsilon} \phi_{0}^{\epsilon} / \sqrt{\epsilon}\right\rangle .
\end{aligned}
$$

In order to evaluate the last limit, the following lemma is essential.

Lemma 9. (a) For each $\psi \in H^{1}[-l, l]$,

$$
\lim _{\epsilon \rightarrow 0}\left\langle\psi, f_{v}^{\epsilon} \phi_{0}^{\epsilon} / \sqrt{\epsilon}\right\rangle=K_{*} J^{\prime}\left(v^{*}\right) \psi\left(x_{a}(l)\right)
$$

(b) As $\epsilon \rightarrow 0$, the function $z^{\epsilon}$ has a limit $z^{*}$ in $H^{1}$ which satisfies

$$
z_{x}^{*}(l) \psi(l)-z_{x}^{*}(-l) \psi(-l)-\left\langle z_{x}^{*}, \psi_{x}\right\rangle+\left\langle B^{*} z^{*}, \psi\right\rangle=K_{*}\left[g_{+}\left(v^{*}\right)-g_{-}\left(v^{*}\right)\right] \psi\left(x_{a}(l)\right),
$$

for $\psi \in H^{1}$ where $B^{*}=g_{*}{ }^{\prime}\left(V_{a}(x)\right)$.

From this lemma, we have

$$
\lim _{\epsilon \rightarrow 0}\left\langle z^{\epsilon}, f_{v}^{\epsilon} \phi_{0}^{\epsilon} / \sqrt{\epsilon}\right\rangle=K_{*} J^{\prime}\left(v^{*}\right) z^{*}\left(x_{a}(l)\right)
$$

If one takes $\psi=V_{a x}$ as a test function in Lemma $8(\mathrm{~b})$, and uses the fact that $V_{a x x x}+$ $B^{*} V_{a x}=0$, then one finds, by using integration by parts, that

$$
z^{*}\left(x_{a}(l)\right)=-K_{*} V_{a x}\left(x_{a}(l)\right)+\frac{z_{x}^{*}(l) V_{a x}(l)-z_{x}^{*}(-l) V_{a x}(-l)}{g_{+}\left(v^{*}\right)-g_{-}\left(v^{*}\right)} .
$$

Therefore, recalling $\lambda_{0}^{*}=-K_{*}^{2} J^{\prime}\left(v^{*}\right) V_{a x}\left(x_{a}(l)\right)$, we have

$$
\hat{\rho}=-K_{*} J^{\prime}\left(v^{*}\right)\left[z_{x}^{*}(l) V_{a x}(l)-z_{x}^{*}(-l) V_{a x}(-l)\right] /\left[g_{+}\left(v^{*}\right)-g_{-}\left(v^{*}\right)\right] .
$$

Since $z_{x}^{*}(l)>0$ and $V_{a x}(l)<0$ are bounded away from zero as $l \rightarrow \infty$, while $z_{x}^{*}(-l)<$ $0, V_{a x}(-l)>0$, go to zero as $l \rightarrow \infty, \hat{\rho}$ is negative for large $l$, say $l>l_{0}$ (see Figure 11). This completes the proof of Lemma 8. 


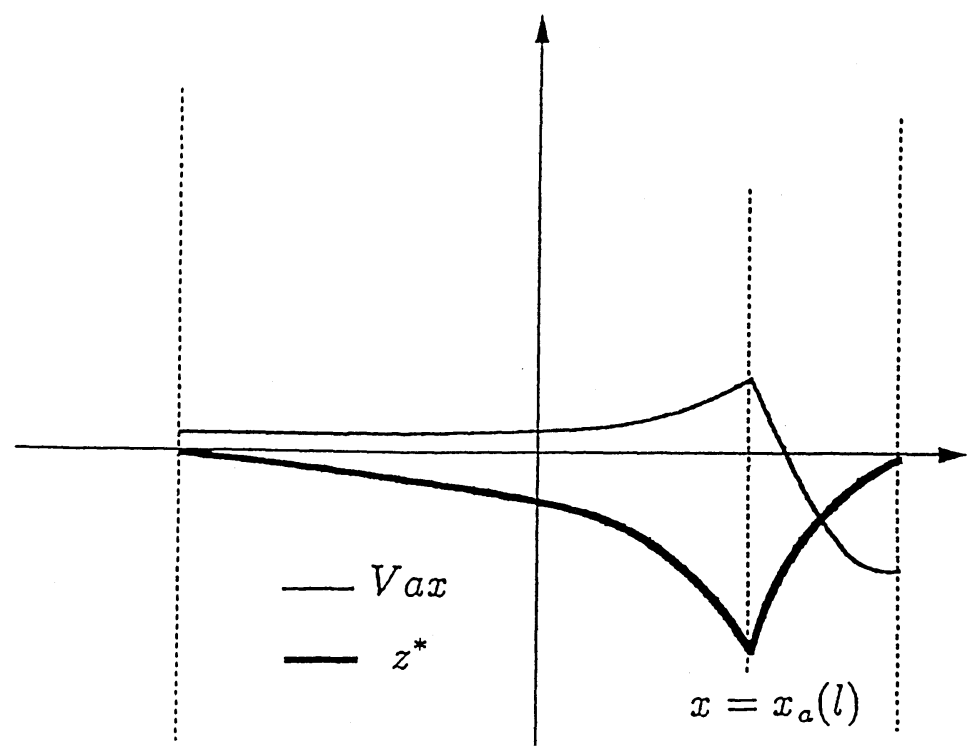

Figure 11. Profiles of $z^{*}$ and $V_{a x}$.

\section{Proof of existence}

We rewrite equation (2.6) as

$$
\mathcal{L}^{\epsilon} W+R^{\epsilon}+F^{\epsilon}(W)=0
$$

where

$$
\mathcal{L}^{\epsilon}=\left(\begin{array}{cc}
L^{\epsilon} & N^{\epsilon} \\
g_{u}^{\epsilon} & M^{\epsilon}
\end{array}\right), \quad W=\left(\begin{array}{c}
u \\
v
\end{array}\right), \quad R^{\epsilon}=\left(\begin{array}{c}
R_{1}^{\epsilon} \\
0
\end{array}\right), \quad F^{\epsilon}(W)=\left(\begin{array}{l}
F_{1}^{\epsilon}(u, v) \\
F_{2}^{\epsilon}(u, v)
\end{array}\right) .
$$

From the main result in the previous section, we know that the operator $\mathcal{L}^{\epsilon}$ has a critical eigenvalue $\rho^{\epsilon}$ with an associated eigenfunction

$$
\Phi^{\epsilon}=\left(\begin{array}{c}
\Phi_{1}^{\epsilon} \\
\Phi_{2}^{\epsilon}
\end{array}\right)=\left(\begin{array}{c}
\sqrt{\epsilon}\left[\phi_{0}^{\epsilon}+K^{\epsilon, \rho^{\epsilon}} \phi_{0}^{\epsilon}\right] \\
-\left(M^{\epsilon}-\rho^{\epsilon}\right)^{-1} g_{u}^{\epsilon} \Phi_{1}^{\epsilon}
\end{array}\right)
$$

and that the remaining part of the spectrum of $\mathcal{L}^{\epsilon}$ is bounded away from zero uniformly in $\epsilon \in\left(0, \epsilon_{0}\right]$. If we denote by $P^{\epsilon}$ the projection onto the span of $\Phi^{\epsilon}$ in $H^{0} \times H^{0}$ and decompose $W$ as $W=a \Phi^{\epsilon}+W_{1}, \Phi^{\epsilon} \perp W_{1}$, then the equation (4.1) is equivalent to

$$
\begin{gathered}
a \rho^{\epsilon}+\frac{1}{\left\|\Phi^{\epsilon}\right\|_{H^{0} \times H^{0}}^{2}}\left\langle R^{\epsilon}+F^{\epsilon}\left(a \Phi^{\epsilon}+W_{1}\right), \Phi^{\epsilon}\right\rangle=0, \\
\mathcal{L}^{\epsilon} W_{1}+\left(I-P^{\epsilon}\right)\left[R^{\epsilon}+F^{\epsilon}\left(a \Phi^{\epsilon}+W_{1}\right)\right]=0 .
\end{gathered}
$$

The last equation (4.3) is solvable in $W_{1}$ as $W_{1}=W_{1}^{\epsilon}(a)$. Substituting this into the equation (4.2), and dividing by $\epsilon$, we obtain a scalar equation $\mathcal{B}(a, \epsilon)=0$ in $a$ and $\epsilon$, where

$$
\mathcal{B}(a, \epsilon)=a \hat{\rho}^{\epsilon}+\frac{1}{\epsilon\left\|\Phi^{\epsilon}\right\|_{H^{0} \times H^{0}}^{2}}\left\langle R^{\epsilon}+F^{\epsilon}\left(a \Phi^{\epsilon}+W_{1}^{\epsilon}(a)\right), \Phi^{\epsilon}\right\rangle
$$


with $\hat{\rho}^{\epsilon}=\rho^{\epsilon} / \epsilon$. The solvability of the scalar equation (4.4) will prove Theorem 2 .

The principal parts of $W_{1}^{\epsilon}(0)$ and $D_{a} W_{1}^{\epsilon}(0)$ are respectively given by

$$
W_{1}^{\epsilon}(0) \approx-\left(\mathcal{L}^{\epsilon}\right)^{-1}\left(I-P^{\epsilon}\right) R^{\epsilon}, \quad D_{a} W_{1}^{\epsilon}(0) \approx-\left(\mathcal{L}^{\epsilon}\right)^{-1}\left(I-P^{\epsilon}\right) D_{w} F^{\epsilon}\left(W_{1}^{\epsilon}(0)\right) \Phi^{\epsilon} .
$$

Since $\left\|R^{\epsilon}\right\|_{H^{0} \times H^{0}}=O\left(\epsilon^{2}\right)$, one easily finds that

$$
\left\|W_{1}^{\epsilon}(0)\right\|_{H_{\epsilon}^{2} \times H^{2}}=O\left(\epsilon^{2}\right), \quad\left\|D_{a} W_{1}^{\epsilon}(0)\right\|_{H_{\epsilon}^{2} \times H^{2}}=O\left(\epsilon^{5 / 2}\right) .
$$

Using these together with $\left\|\Phi^{\epsilon}\right\|_{H^{0} \times H^{0}}=O(\sqrt{\epsilon})$, one can compute the following limits:

$$
\lim _{\epsilon \rightarrow 0} \mathcal{B}(0, \epsilon)=0, \quad \lim _{\epsilon \rightarrow 0} D_{a} \mathcal{B}(0, \epsilon)=\rho^{*}<0 .
$$

Therefore, using the implicit function theorem, one obtains the solvability in $a$ of the equation $\mathcal{B}(a, \epsilon)=0$ as $a=a(\epsilon)$ with $a(\epsilon)=O(\sqrt{\epsilon})$.

If we denote the $u$ - and $v$-components of $a(\epsilon) \Phi^{\epsilon}+W_{1}^{\epsilon}(a(\epsilon))$ by $u_{1}^{\epsilon}$ and $v_{1}^{\epsilon}$, respectively, then the desired solution $\left(u_{s}^{\epsilon}, v_{s}^{\epsilon}\right)$ in Theorem 2 is given by

$$
u_{s}^{\epsilon}=U^{\epsilon}+D_{v} U^{\epsilon} v_{1}^{\epsilon}+u_{1}^{\epsilon}, \quad v_{s}^{\epsilon}=V^{\epsilon}+v_{1}^{\epsilon} .
$$

This completes the proof of Theorem 2 for the symmetric solution. The proof of Theorem 2 for the antisymmetric solution is exactly the same as above by using the results of Subsection 3.2, and hence will not be repeated.

\section{Stability analysis}

In this section, we analyse the stability property of the symmetric solution obtained in the previous sections. The arguments here are almost identical to those of Section 3. We consider the eigenvalue problem

$$
\left(\begin{array}{cc}
\epsilon^{2} d^{2} / d x^{2}+f_{u}^{\epsilon} & f_{v}^{\epsilon} \\
g_{u}^{\epsilon} & d^{2} / d x^{2}+g_{v}^{\epsilon}
\end{array}\right)\left(\begin{array}{l}
u \\
v
\end{array}\right)=\rho\left(\begin{array}{l}
u \\
v
\end{array}\right),
$$

with the boundary conditions $u( \pm l)=0, v( \pm l)=0$, where $f_{u}^{\epsilon}$, etc., are evaluated at $(u, v)=\left(u_{s}^{\epsilon}, v_{s}^{\epsilon}\right)$. Because the functions $f_{u}^{\epsilon}$, etc., are even, all the eigenfunctions are either even (symmetric) or odd (antisymmetric). Therefore one is allowed to consider the problem on the half interval $[-l, 0]$ by examining equation (5.1) with the two sets of boundary conditions (DN) and (DD) which appeared in Section 3.1.

As was done for the existence proof, we change variables in the above equation by

$$
u=D_{v} U^{\epsilon} z+w, \quad v=z .
$$

The resulting equation for $(w, z)$ is

$$
\left(\begin{array}{ll}
L^{\epsilon} & N^{\epsilon} \\
g_{u}^{\epsilon} & M^{\epsilon}
\end{array}\right)\left(\begin{array}{l}
w \\
z
\end{array}\right)=\rho\left(\begin{array}{cc}
1 & D_{v} U^{\epsilon} \\
0 & 1
\end{array}\right)\left(\begin{array}{l}
w \\
z
\end{array}\right)
$$

where the operators $L^{\epsilon}, M^{\epsilon}$, and $N^{\epsilon}$ are given by the same formulae as in Section 2, but with the derivatives of $f, g$ evaluated at $(u, v)=\left(u_{s}^{\epsilon}, v_{s}^{\epsilon}\right)$. All the results in Section 3 concerning these operators are valid for those in this section. Therefore the second equation in (5.2) can be solved in $z$, and substituting this into the first, we obtain

$$
\begin{gathered}
\alpha\left(\lambda_{0}^{\epsilon}-\rho\right)-\left\langle\left(M^{\epsilon}-\rho\right)^{-1} g_{u}^{\epsilon}\left(\alpha \phi_{0}^{\epsilon}+w_{1}\right),\left(N_{*}^{\epsilon}-\rho D_{v} U^{\epsilon}\right) \phi_{0}^{\epsilon}\right\rangle=0, \\
\left(L^{\epsilon}-\rho\right) w_{1}-Q\left(N^{\epsilon}-\rho D_{v} U^{\epsilon}\right)\left(M^{\epsilon}-\rho\right)^{-1} g_{u}^{\epsilon} w_{1}=\alpha Q\left(N^{\epsilon}-\rho D_{v} U^{\epsilon}\right)\left(M^{\epsilon}-\rho\right)^{-1} g_{u}^{\epsilon} \phi_{0}^{\epsilon} .
\end{gathered}
$$


Since $D_{v} U^{\epsilon}$ is bounded by a constant independent of $\epsilon$, there is a positive constant $\delta$ such that if $|\rho|<\delta$ then $^{1}$ the second equation in the above is solvable in $w_{1}$ as $w_{1}=\alpha K^{\epsilon, \rho} \phi_{0}^{\epsilon}$. Substituting this into the first equation, we obtain

$$
\begin{gathered}
\left(\lambda_{0}^{\epsilon}-\rho\right)\left[1-\left\langle\left(M^{\epsilon}-\rho\right)^{-1} g_{u}^{\epsilon}\left(\phi_{0}^{\epsilon}+K^{\epsilon, \rho} \phi_{0}^{\epsilon}\right), D_{v} U^{\epsilon} \phi_{0}^{\epsilon}\right\rangle\right] \\
=\left\langle\left(M^{\epsilon}-\rho\right)^{-1} g_{u}^{\epsilon}\left(\phi_{0}^{\epsilon}+K^{\epsilon, \rho} \phi_{0}^{\epsilon}\right), f_{v}^{\epsilon} \phi_{0}^{\epsilon}\right\rangle .
\end{gathered}
$$

Arguing as in Section 3, one finds that $\rho=O(\epsilon)$. Therefore, with $\rho=\epsilon \hat{\rho}_{j}+o(\epsilon)$, $j=s, a$, one finds

$$
\lambda_{0}^{*}-\rho_{j}^{*}=\lim _{\epsilon \rightarrow 0} \frac{1}{\epsilon}\left\langle\left(M^{\epsilon}-\left(\epsilon \rho^{*}+o(\epsilon)\right)\right)^{-1} g_{u}^{\epsilon} \phi_{0}^{\epsilon}, f_{v}^{\epsilon} \phi_{0}^{\epsilon}\right\rangle, \quad j=s, a .
$$

The symmetric case $(j=s)$ is exactly the same as in Section 3 , therefore

$$
\hat{\rho}_{s}=\frac{K_{*} J^{\prime}\left(v^{*}\right)\left[z_{x}^{*}(-l) V_{s x}(-l)+z^{*}(0) V_{s x x}(0)\right]}{\left[g_{+}\left(v^{*}\right)-g_{-}\left(v^{*}\right)\right]}<0 .
$$

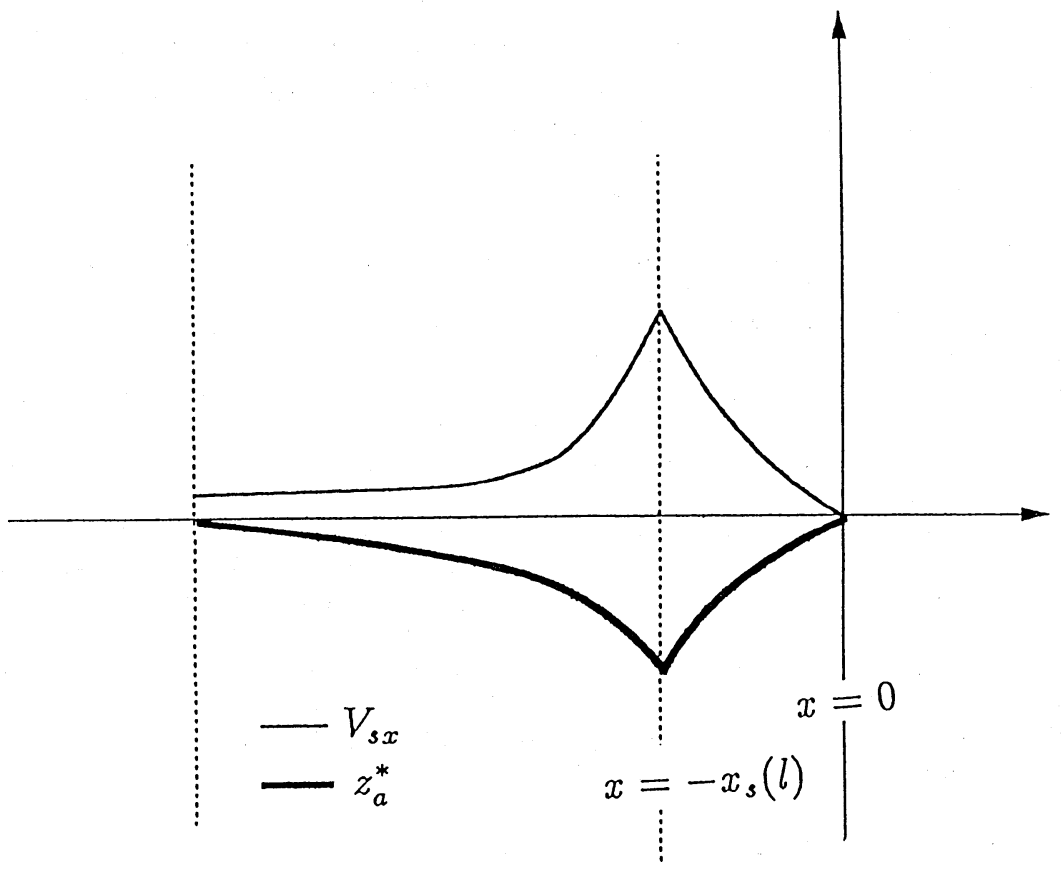

Figure 12. Profiles of $z_{a}^{*}$ and $V_{s x}$.

In order to examine the antisymmetric case $(j=a)$, we need the following lemma.

Lemma 10. (a) For each $\psi \in H^{1}$,

$$
\lim _{\epsilon \rightarrow 0}\left\langle\psi, f_{v}^{\epsilon} \phi_{0}^{\epsilon} / \sqrt{\epsilon}\right\rangle=K_{*} J^{\prime}\left(v^{*}\right) \psi\left(-x_{s}(l)\right) .
$$

(b) Let $z_{a}^{\epsilon}=\left(M^{\epsilon}\right)^{-1} g_{u}^{\epsilon} \phi_{0}^{\epsilon} / \sqrt{\epsilon}$ with the boundary condition (DD). As $\epsilon \rightarrow 0$, the function $z_{a}^{\epsilon}$ has a limit $z_{a}^{*}$ in $H^{1}$ which satisfies

$z_{a x}^{*}(0) \psi(0)-z_{a x}^{*}(-l) \psi(-l)-\left\langle z_{a x}^{*}, \psi_{x}\right\rangle+\left\langle B^{*} z_{a}^{*}, \psi\right\rangle=K_{*}\left[g_{+}\left(v^{*}\right)-g_{-}\left(v^{*}\right)\right] \psi\left(-x_{s}(l)\right)$,

${ }^{1}$ If $|\rho| \geq \delta$, then an argument in Nishiura and Fujii [1987] shows that $\Re \rho<-\rho_{1}$ for some $\rho_{1}>0$. 
for $\psi \in H^{1}$, where $B^{*}=g_{*}{ }^{\prime}\left(V_{s}(x)\right)$.

From this lemma, it follows that

$$
\lambda_{0}^{*}-\hat{\rho}_{a}=\lim _{\epsilon \rightarrow 0}\left\langle z_{a}^{\epsilon}, f_{v}^{\epsilon} \phi_{0}^{\epsilon} / \sqrt{\epsilon}\right\rangle=K_{*} J^{\prime}\left(v^{*}\right) z_{a}^{*}\left(-x_{s}(l)\right) .
$$

If one takes $\psi=V_{s x}$ as a test function in the above, one finds

$$
z_{a}^{*}\left(-x_{s}(l)\right)=-K_{*} V_{s x}\left(-x_{s}(l)\right)-\frac{z_{a x}^{*}(-l) V_{s x}(-l)}{g_{+}\left(v^{*}\right)-g_{-}\left(v^{*}\right)} .
$$

Therefore

$$
\hat{\rho}_{a}=K_{*} J^{\prime}\left(v^{*}\right) \frac{z_{a x}^{*}(-l) V_{s x}(-l)}{g_{+}\left(v^{*}\right)-g_{-}\left(v^{*}\right)},
$$

which is positive since $J^{\prime}\left(v^{*}\right)<0, z_{a}^{*}(-l)<0$. This completes the proof of Theorem 3(i) (see Figure 12). The proof of Theorem 3(ii) is the same as that of Lemma 7 in Subsection 3.2.

\section{Acknowledgment}

The author is grateful to Professors M. Mimura and T. Tsujikawa for their interest in this work and helpful comments. He is also thankful to Mr. Hideo Sakaguchi for performing a numerical simulation for this work.

\section{References}

1. J. Elezgaray and A. Arneodo, Modeling reaction-diffusion pattern formation in the Couette flow reactor, J. Chem. Phys. 95(1) (1991), 323-350.

2. P. C. Fife, Boundary and interior transition layer phenomena for pairs of second-order differential equations, J. Math. Anal. Appl., 54 (1976), 497-521.

3. P. Gray, et al., Spatial inhomogenieties and transient behavior in chemical kinetics, Proceedings in Nonlinear Science, Manchester University Press, 1990.

4. J. K. Hale and K. Sakamoto, Existence and stability of transition layers, Japan J. Appl. Math., 5 (1988), 367-405.

5. M. Ito, A remark on singular perturbation methods, Hiroshima Math. J., 14 (1985), 619-629.

6. M. Mimura, M. Tabata, and Y. Hosono, Multiple solutions of two-point boundary value problems of Neumann type with a small parameter, SIAM J. Math. Anal., 11 (1980), 613-631.

7. J. D. Murray, Mathematical Biology, Springer-Verlag, 1989.

8. Y. Nishiura and H. Fujii, Stability of singularly perturbed solutions to systems of reactiondiffusion equations, SIAM J. Math. Anal., 18 (1987), 1726-1770.

9. K. Sakamoto, Construction and stability analysis of transition layer solutions in reactiondiffusion systems, Tohoku Math. J., 42 (1990), 17-44.

Department of Mathematics, Hiroshima University, Higashi-Hiroshima, Japan 